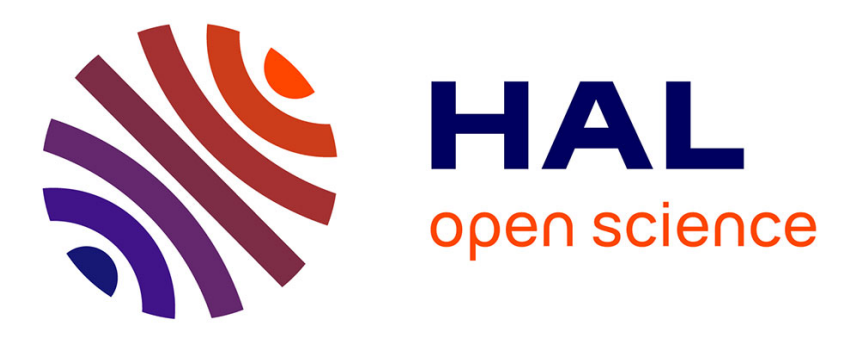

\title{
Experimental and theoretical studies of tetramethoxy-p-benzoquinone: infrared spectra, structural and lithium insertion properties
}

Gaëtan Bonnard, Anne-Lise Barrès, Yann Danten, Damian G. Allis, Olivier Mentre, Daniele Tomerini, Carlo Gatti, Ekaterina I. Izgorodina, Philippe Poizot, Christine Frayret

\section{To cite this version:}

Gaëtan Bonnard, Anne-Lise Barrès, Yann Danten, Damian G. Allis, Olivier Mentre, et al.. Experimental and theoretical studies of tetramethoxy-p-benzoquinone: infrared spectra, structural and lithium insertion properties. RSC Advances, 2013, 41 (3), pp.19081-19096. 10.1039/C3RA41974F . hal-00952928

\section{HAL Id: hal-00952928 \\ https://hal.science/hal-00952928}

Submitted on 1 Mar 2014

HAL is a multi-disciplinary open access archive for the deposit and dissemination of scientific research documents, whether they are published or not. The documents may come from teaching and research institutions in France or abroad, or from public or private research centers.
L'archive ouverte pluridisciplinaire HAL, est destinée au dépôt et à la diffusion de documents scientifiques de niveau recherche, publiés ou non, émanant des établissements d'enseignement et de recherche français ou étrangers, des laboratoires publics ou privés. 
Cite this: RSC Advances, 2013, 3, 19081

Received 22nd April 2013, Accepted 29th July 2013

DOI: $10.1039 / \mathrm{c} 3 \mathrm{ra} 41974 \mathrm{f}$ www.rsc.org/advances

\section{Experimental and theoretical studies of tetramethoxy- $p$-benzoquinone: infrared spectra, structural and lithium insertion properties $\dagger$}

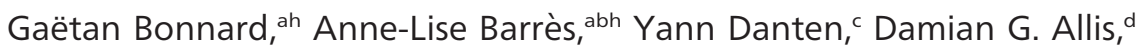

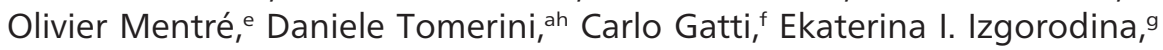 \\ Philippe Poizot ${ }^{\text {abh }}$ and Christine Frayret*ah
}

\begin{abstract}
In the search for low-polluting electrode materials for batteries, the use of redox-active organic compounds represents a promising alternative to conventional metal-based systems. In this article we report a combined experimental and theoretical study of tetramethoxy-p-benzoquinone (TMQ). In carbonate-based electrolytes, electrochemical behaviour of this compound is characterized by a reversible insertion process located at approximately $2.85 \mathrm{~V} \mathrm{vs.} \mathrm{Li}^{+} / \mathrm{Li}^{0}$. This relatively high potential reactivity, coupled with our effort to develop computational methodologies in the field of organic electrode materials, prompted us to complement these experimental data with theoretical studies performed using density functional theory (DFT). Single crystals of TMQ were synthesized and thoroughly characterized showing that this quinonic species crystallised in the $P 2_{1} / n$ space group. The experimental crystal structure of TMQ was then used to assess various DFT methods. The structural features and vibrational spectra were thus predicted by using as a whole five common density functionals (PBE, LDA, revPBE, PBEsol, B3PW91) with and without a semi-empirical correction to account for the van der Waals interactions using either Grimme's (DFT-D2) or Tkatchenko-Scheffler (TS) scheme. The most reliable combination of the DFT functional and the explicit dispersion correction was chosen to study the Li-intercalated molecular crystal (LiTMQ) with the view of indentifying Li insertion sites. A very close agreement with the experiment was found for the average voltage by using the most stable relaxed hypothetical LiTMQ structure. Additionally, a comparison of vibrational spectra gained either for TMQ molecule and its dimer in gas phase or through periodic calculation was undertaken with respect to the experimentally measured infrared spectra. The topological features of the bonds were also investigated in conjunction with estimates of net atomic charges to gain insight into the effect of chemical bonding and intermolecular interaction on $\mathrm{Li}$ intercalation. Finally, $\pi$-electron delocalization of both quinone and alkali salts of $p$-semiquinone were determined using the Harmonic Oscillator model of Aromaticity (HOMA) or aromatic fluctuation index (FLU) calculations.
\end{abstract}

\section{Introduction}

The market for lithium-ion batteries (LiBs) is considerable in portable electronic devices due to their low weight and high energy density. Today LiBs are widely used as secondary batteries from low- to high-power applications such as electric vehicles, for which mass production is expected in the near future. However, the combination of scarcity of rare metal oxides in the earth's crust for production of positive electrodes in these batteries and high temperatures required for their synthesis result in both high environmental impact and total cost if current LiB approaches are retained.

Due to such concerns, our group and others ${ }^{1-15}$ have recently focused on the development of organic crystalline cathodes and anodes with the hope of their utilization in 
metal-free electrodes to be used in a new generation of LiBs with lower carbon footprints and simplified recycling requirements. The construction of potentially sustainable and costeffective rechargeable lithium batteries from such materials might even be possible in some cases.

Here, we report a combined experimental and theoretical study aimed at providing a contribution to the ultimate goal of the prediction of structural and electrochemical properties of such organic crystalline materials. In this work tetramethoxy$p$-benzoquinone (TMQ) was chosen as an example of such a material, which was studied experimentally by means of electrochemical techniques and computationally using density functional theory (DFT). This combined approach was employed specifically to interpret vibrational features of TMQ and predict structural and electrochemical properties of the lithiated-phase crystal that results from lithium intercalation in the solid-state TMQ (i.e. reduction of one carbonyl group per molecule leading to the corresponding $p$-semiquinone anion radical), as no reliable X-ray diffraction data are currently available for the LiTMQ (hereafter denoted as LiTMQ for the sake of simplicity) crystal.

From the theoretical viewpoint, this work will focus on (i) geometry and vibrational spectra properties for both TMQ molecule and its dimer in gas phase as well as corresponding bulk crystal (Fig. 1) (ii) the prediction of a crystal structure for the intercalated material (LiTMQ) through identification of the most suited model among different hypotheses from the viewpoint of lithium insertion sites and (iii) the estimation of the calculated intercalation potential and comparison to the experimental value. The prerequisite for such a study is to define the most suited computational treatment for this particular material (TMQ). With this aim, the relative ability to reproduce structural features was considered for the whole set of selected methodologies. For the solid-state studies, we compared the performance of several DFT approaches using different exchange-correlation (XC) functionals (either nonhybrid: LDA, ${ }^{16}$ GGA (PBE) ${ }^{17}$ revPBE, ${ }^{18}$ PBEsol $^{19}$ or hybrid: B3PW91 ${ }^{20}$ ) with and without an explicit correction for dispersion forces developed by S. Grimme (DFT-D2) ${ }^{21}$ or A. Tkatchenko and M. Scheffler (TS). ${ }^{22}$ Topological features of charge densities issued from our theoretical analysis on the two crystal forms (TMQ and LiTMQ) were then analyzed through bond critical point (BCP) parameters ${ }^{23}$ with the view of gaining insight on the chemical bonding transformation induced by the intercalation of lithium. A comparison of vibrational spectra gained either for TMQ molecule and its dimer in gas phase or through periodic calculation was undertaken with respect to the experimentally measured infrared spectra.

\section{Methods}

TMQ was synthesized from chloranil through a single-step reaction according to a previously described synthesis. ${ }^{24}$ At the
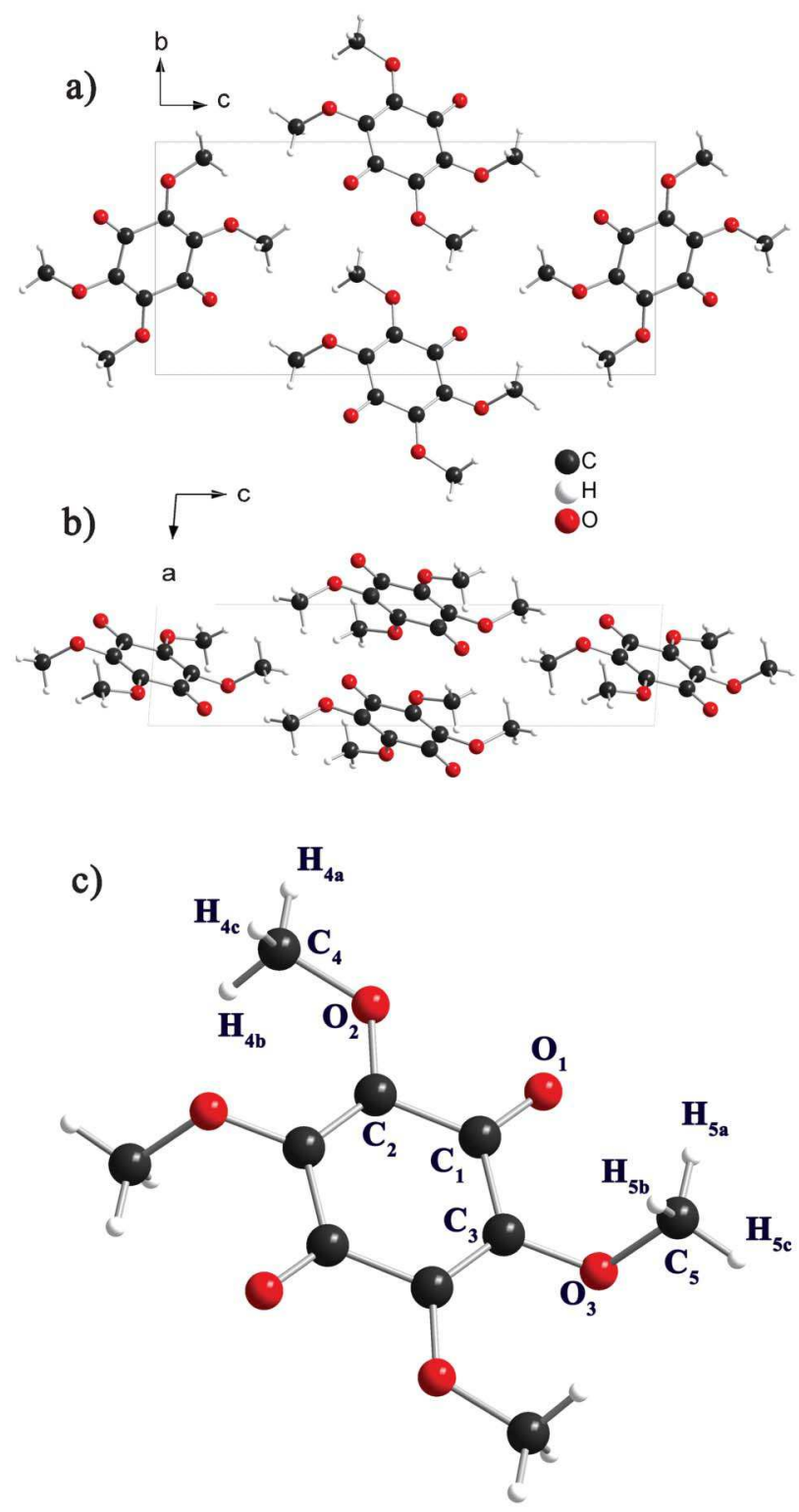

Fig. 1 Crystal structure of the TMQ compound in different projections. (Oxygen positions are in red, carbon positions in dark gray, and hydrogen positions in white). (a) View along the $a$ axis; (b) view along the $b$ axis; (c) atom labels with the molecule view along the $a$ axis.

end of the synthesis, TMQ was recovered as single crystals without further recrystallization.

Single crystal XRD measurements were carried out at both RT and $100 \mathrm{~K}$ using a Bruker X8 Apex2 CCD4K (Mo-K $\alpha$ radiation, $\lambda=0.71073 \AA$ ) equipped with an Oxford Cryostream cooling system. Absorption corrections were performed using the multiscan SADABS program. ${ }^{25}$ Structures were solved by direct methods using SHELX ${ }^{26}$ and least-squares refined with the JANA2006 software package. ${ }^{27}$ Crystallographic data for the TMQ phase at both RT and $100 \mathrm{~K}$ are listed in Table 1.

Electrochemical data were obtained by using a Swagelok ${ }^{\mathbb{R}}$ type cell, a Li metal disc as negative electrode, and a fiberglass separator soaked with a molar $\mathrm{LiPF}_{6}$ solution (in ethylene 
Table 1 Crystallographic data collection and structure refinement details at 100 $\mathrm{K}$ and room temperature for the TMQ compound

\begin{tabular}{lll}
\hline Crystal data & $100 \mathrm{~K}$ & Room temperature \\
\hline Formula & $\mathrm{C}_{10} \mathrm{H}_{12} \mathrm{O}_{6}$ & $\mathrm{C}_{10} \mathrm{H}_{12} \mathrm{O}_{6}$ \\
$T / \mathrm{K}$ & 100 & 298 \\
$M_{\mathrm{r}} / \mathrm{g} \mathrm{mol}^{-1}$ & 228.2 & 228.2 \\
$\mathrm{Crystal}^{-1}$ system & Monoclinic & Monoclinic \\
Space group & $P 2_{1} / n$ & $P 2_{1} / n$ \\
$a / \AA$ & $3.9137(2)$ & $4.0075(2)$ \\
$b / \AA$ & $7.7232(4)$ & $7.7425(4)$ \\
$c / \AA$ & $16.6264(9)$ & $16.7724(6)$ \\
$\beta\left({ }^{\circ}\right)$ & $93.410(3)$ & $94.112(3)$ \\
$V / \AA^{3}$ & $501.66(5)$ & $519.08(4)$ \\
$Z$ & 2 & 2 \\
$D_{\text {calcd }} / \mathrm{mg} \mathrm{m}^{-3}$ & 1.511 & 1.460 \\
$F(000)$ & 220 & 296 \\
$2 \theta_{\text {max }}\left({ }^{\circ}\right)$ & 78.72 & 55.34 \\
Reflections measured & 20724 & 5266 \\
Reflections unique $\left(\mathrm{R}_{\text {int }}\right)$ & $2892(0.0257)$ & $1212(0.0480)$ \\
Number of parameters & 75 & 76 \\
$\mathrm{GOF}(S)$ & 1.167 & 1.025 \\
$R_{1}, \mathrm{w} R_{2}[I>2 \sigma(I)]$ & $0.0341,0.1337$ & $0.0469,0.1099$ \\
$R_{1}, \mathrm{w} R_{2}($ all data) & $0.0397,0.1411$ & $0.0966,0.1298$ \\
$\Delta(\rho) / \mathrm{e} \mathrm{A}^{-3}$ & $0.728,-0.284$ & $0.193,-0.182$ \\
& & \\
\hline
\end{tabular}

carbonate : dimethyl carbonate/1 : 1 in volume ratio) as the electrolyte as previously described. ${ }^{6}$ The positive electrode was prepared without a binder by mixing TMQ powder with 33 weight \% carbon SP (in total mass). The cell was then cycled by using a galvanostatic intermittent titration technique (GITT) within a potential window ranging from 3.5 to $2.2 \mathrm{Vvs} . \mathrm{Li}^{+} / \mathrm{Li}^{0}$ (VMP system, Biologic S.A., Claix, France). Experimentally, a constant current equivalent to a cycling rate of one $\mathrm{Li}^{+}$ exchanged in $10 \mathrm{~h}$ was applied during $2 \mathrm{~h}$ (i.e., $\Delta \mathrm{x}=0.2$ ), followed by a relaxation period of $1 \mathrm{~h}$ at zero current.

Infrared (IR) spectra of polycrystalline TMQ were measured on a Bruker VERTEX 70V FT-IR spectrometer equipped with a deuterated triglycylsulphate (DGTS) detector. Pellets were prepared by thoroughly mixing the organic compound with spectroscopic-grade potassium bromide (99+\% from Acros Organics). The range of measurement was $400-4000 \mathrm{~cm}^{-1}$ and the resolution was $4 \mathrm{~cm}^{-1}$.

The geometries of the TMQ isolated molecule and corresponding dimers were optimized using the Gaussian09 $\operatorname{program}^{28}$ at the B3PW91/6-311+G(2d,p) level of theory. The vibrational analysis was carried out for the optimized structures of the molecular and dimeric forms of TMQ within the standard Wilson FG matrix formalism (harmonic normal modes) implemented in the Gaussian09 package. In this framework, the predicted harmonic vibrational frequencies are usually overestimated compared to the experimental ones due to omission of anharmonicity effects. To improve predicted spectra, scaling factors for fundamental vibrational energies have been reported for different B3-DFT functionals. The scaling factor optimized for the B3PW91 functional with the $6-311+\mathrm{G}(2 \mathrm{~d}, \mathrm{p})$ basis set is $0.9631 .^{29}$

The optimized structures of the two bulk crystals (TMQ and LiTMQ) were obtained from the VASP ${ }^{30}$ CRYSTAL09 ${ }^{31,32}$ and CASTEP $^{33}$ packages by taking into account the empirical dispersion of Grimme ${ }^{21}$ or that of Tkatchenko-Scheffler (TS). ${ }^{22}$ Calculations were carried out at the PBE-D and B3PW91-D levels of theory. For Grimme's correction, inclusion of modified vdW radii proposed by Civalleri et $a .^{34}$ and additional adjustment of the $\mathrm{s}_{6}$ values suggested by King et $a l^{35}$ have been considered in the study, labeled PBE-D* and corr-PBE-D*_0.52, repsectively. ${ }^{6}$ Optimal $\mathrm{s}_{6}$ values were identified to be 0.52 and 0.70 for the TMQ phase at RT and at $100 \mathrm{~K}$, respectively. Classical XC functionals were tested using the VASP package only. Concerning dispersion-corrected DFT, the results obtained from the PBE-D, PBE-D* and corr-PBE-D*-x.xx ( $\mathrm{x} . \mathrm{xx}$ corresponding to the $\mathrm{s}_{6}$ value) VASP calculations were compared to the CRYSTAL (corr-PBE-D*_x.xx/6-31G** and corrB3PW91-x.xx/6-31G**) and CASTEP (PBE-TS) treatments, which correspond respectively to plane-wave/pseudopotential and LCAO/all electron methods. As a point of clarification, the CRYSTAL calculations are always listed with their associated Gaussian-type basis set, while VASP calculations are listed with the functional only. Following the investigations of TMQ, LiTMQ computations were undertaken with the most suited methodology evidenced from the RT calculations of the unlithiated TMQ phase through direct comparison between theoretical and experimental data.

For both geometry optimised TMQ and LiTMQ phases, we applied the atoms-in-molecules (AIM) theory, which was developed by Bader and co-workers. Such partitioning of the electron density allows for the evaluation of atomic charges, ${ }^{36-40}$ which can be useful for estimation of charge transfer between ions. It also describes the molecular topology through the determination of Bond Critical Points (BCPs) between two atoms. At each BCP, several properties may be calculated, such as the electron density $(\rho)$ and the Laplacian field $\nabla^{2} \rho(\mathrm{r})$. These properties are related to the charge distribution and to the extent of electron sharing between the linked atoms, allowing for a classification of the interaction as shared (covalent) or closed-shell (ionic/hydrogen bond) when the electron density is either concentrated or depleted, respectively. BCPs along with net atomic charges (NACs) for the phases TMQ and LiTMQ were analyzed through the $\mathrm{AIM}^{23}$ theory by using the TOPOND ${ }^{41}$ program. Finally, various $\pi$-electron delocalization such as $\mathrm{HOMA}^{42,43}$ and $\mathrm{FLU}^{44,45}$ were estimated for both molecular and crystalline states. Further details of all computations are available from the electronic supplementary information (ESI $\dagger$ ).

\section{Results and discussion}

\subsection{Structural features of the crystalline TMQ phase}

The TMQ crystal structure has already been reported twice in the literature. ${ }^{46,47}$ Both structures, which crystallized in the monoclinic $P 2_{1} / c$ and $P 2_{1} / n$ space groups, proved to be equivalent, since a simple relationship between the two forms could be evidenced as $\vec{a}_{1}=\vec{a}_{2}, \vec{b}_{1}=-b_{2}, \vec{c}_{1}=-\vec{c}_{2}-\vec{a}_{2}$, where 1 and 2 refer to the structures described in the $P 2_{1} / c$ and $P 2_{1} / n$ space groups, respectively. 

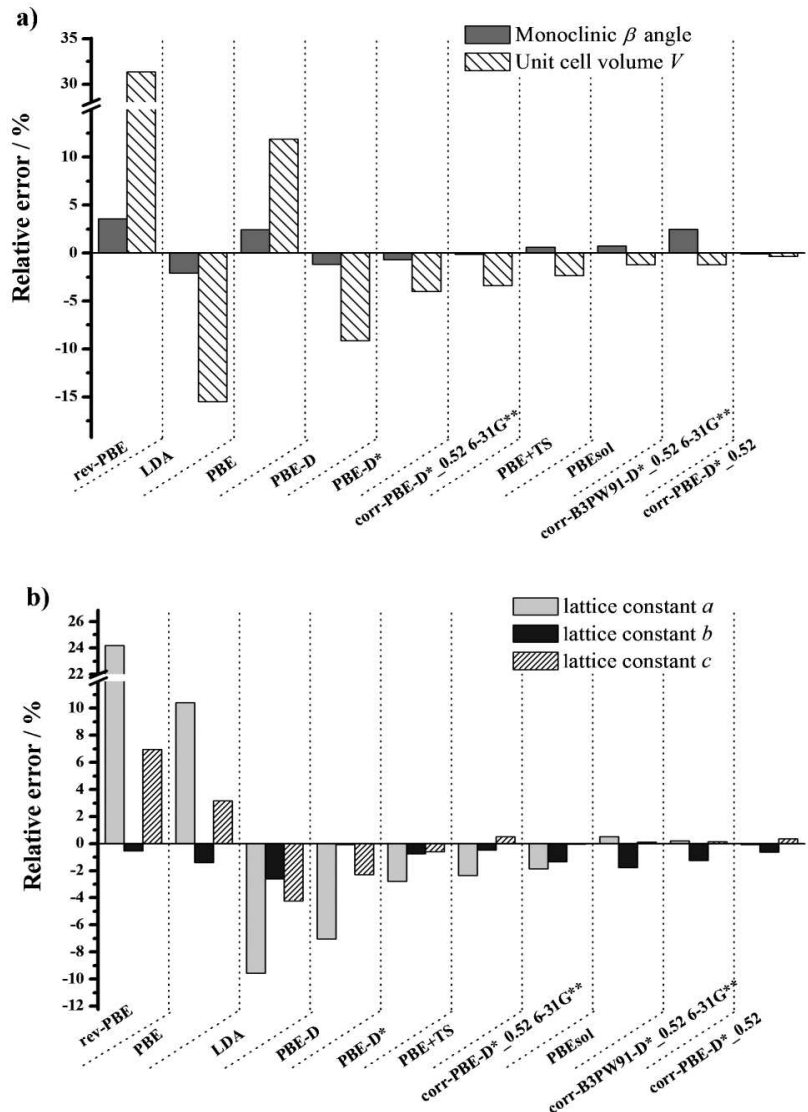

Fig. 2 Relative errors (\%) of DFT or DFT-D calculated values to experiment: (a) for the unit-cell volume $V$ and monoclinic angle $\beta$; (b) for the optimized lattice parameters, $a, b$ and $c$.

In this work, single crystal X-ray diffraction measurements were performed at both RT and $100 \mathrm{~K}$ in order to rule out any phase transition possibility. Structure resolution showed that the phase was temperature-independent (absence of phase transition) and corresponded to the one previously described 46,47 (with only slight unit cell parameter modifications). TMQ crystallized in the monoclinic space group $P 2_{1} / n$ with the following unit cell parameters: $a=4.0075(2) \AA, b=7.7425(4) \AA$, $c=16.7724(6) \AA, \beta=94.112(3)^{\circ}, V=519.08(4) \AA^{3}$ and $Z=2$. A representation of the structure is shown in Fig. 1. TMQ molecules are stacked along the $a$-axis (Fig. S2a, ESI $\dagger$ ), defining a onedimensional columnar structure with an inter-plane distance of $3.46 \AA$ between two adjacent molecules and an offset of $2.02 \AA$. Due to the molecule orientations, dispersion effects may also arise along the $b$ and $c$ axes to a lower extent.

In Fig. 2 and Table S1 of ESI, $\dagger$ we present the results of the optimized crystals of TMQ at RT using different XC approximations as well as various dispersion corrections. One observes strong differences in the unit cell volume and lattice parameters for TMQ across the used XC functionals and dispersion-corrected functionals such as PBE-D and PBE-TS. Values of the monoclinic angle $\beta$ only diverge from the experimental value from 1 to 4 degrees, while for the lattice constants $a, b$, and $c$, the differences of up to $24 \%$ are found. Among the XC functionals used, the PBEsol compares best to the parameters of the experimental crystal structure. PBEsol lattice constants are only 1-3\% smaller than the corresponding experimental values, whereas revPBE and LDA give very poor results. Taking generalised gradient corrections into account for XC (PBE) leads to an $a$-axis length overestimation of approximately the same amount as the underestimation found for the LDA functional $(\sim 10 \%)$. On the other hand, considering this functional, slight contraction and expansion of $\sim-2 \%$ and $+3 \%$ are observed along the $b$ - and $c$-axes, respectively.

Beyond the structural changes resulting from the $\mathrm{XC}$ methodological approximations, the intermolecular interaction is not equally well accounted for within the three approximations in the PBE-D treatment, which is in agreement with previous studies. ${ }^{6,48,49}$ Whereas the underestimation of long-range electron correlation in PBE yields incorrect lattice parameters characterized especially by a strong overestimation of the $a$-axis as already stated, the PBE-D functional overcompensates such effects and leads to a contraction of $-7 \%$ of the $a$-axis with respect to the experiment. This overcompensation for dispersion forces when using the original Grimme's parameters for the dispersion correction was noticed previously in our works for the structural optimizations of other molecular crystals. ${ }^{6,48,49}$ The proposed correction by Civalleri et al. (PBE-D*), corresponding to the modification of the van der Waals radii, improves the description of the dispersion interaction, leading to a better reproduction of the lattice parameters. Indeed, the underestimation of the $a$-lattice parameter is reduced by half when using PBE-D* $(\sim-3 \%)$. As already established in our previous study of the tetrahydrated dilithium salt of 3,6-dihydroxy-2,5dimethoxy- $p$-benzoquinone $\left(\mathrm{Li}_{2} \mathrm{DHDMQ} \cdot 4 \mathrm{H}_{2} \mathrm{O}\right),{ }^{6}$ the adjustment of the $\mathrm{s}_{6}$ parameter provided further improvement to the theoretical lattice parameter values. The dimensions of the fully-optimized unit cell matched better when replacing the original $s_{6}$ value of 0.75 with 0.52 . In the current study among the used corr-PBE-D*_0.52/6-31G**, corr-B3PW91_0.52/6$31 \mathrm{G}^{* *}$ and corr-PBE-D*_0.52 combinations, the corr-PBE$\mathrm{D}^{*}$ _0.52 functional appeared to provide the smallest deviations from experiment $(|\Delta x| / x<1 \%$ for all lattice parameters), with the choice of basis set being less significant compared to the choice of the dispersion correction. Change in volume using PBE-TS is less satisfactory than that for corr-PBE$\mathrm{D}^{*} \_0.52$ (with an underestimation by $\sim-2.4 \%$ ) and is also accompanied by poorer geometry parameters compared to the PBEsol results. On the other hand, a large difference is noticed between the inter-plane distance, $d$, calculated for the whole series of the dispersion-corrected methods and original PBE, with a relative error for the latter exceeding $+13 \%$ (Fig. 3a). This indicates that the interaction between TMQ layers are dominated by vdW interactions. However, the extent of discrepancy is much more pronounced for the PBEsol XC functional $(\sim+2 \%)$. The results obtained from the original parameters of Grimme's in PBE-D again exhibit a noticeable overcompensation with a relative error of $\sim-7 \%$. Such effects become more pronounced when taking into account the Civalleri's modifications. Nearly negligible discrepancies with the experiment are observed for corr-PBE-D*_0.52, PBE-TS and corr-B3PW91-D*_0.52/6-31G**. Concerning offset values, dis- 

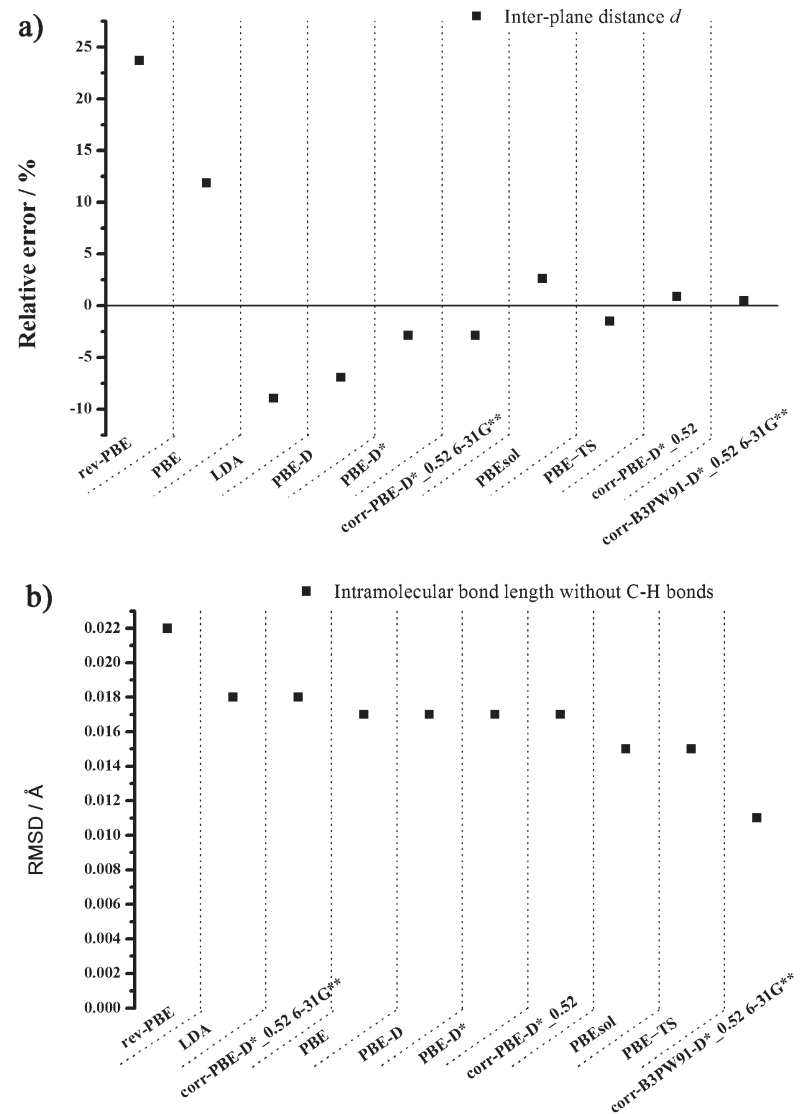

Fig. 3 (a) Relative errors (\%) of DFT or DFT-D calculated values to experiment for the inter-plane distance $d$. (b) RMSD values with respect to experimental data for the intramolecular bond lengths and intermolecular distances without taking into account covalent bonds involving either $\mathrm{H}$ atoms or $\mathrm{H}$-bonding interactions.

crepancies with the experimental data are lower than 5\% (with the largest deviation being found for the PBE-TS combination) in the case of the best performing dispersion corrected methods. Particularly low differences are observed for the corr-PBE-D*_0.52/6-31G** (+0.99\%) and corr-B3PW91_0.52/6$31 G^{* *}(-0.50 \%)$ CRYSTAL calculations.

The quality of the predicted intramolecular structural parameters is reported in terms of root mean-squared deviations (RMSDs) of bond lengths between the calculated structures and the experimental structures. The RMSDs estimated for the whole set of $\mathrm{C}-\mathrm{C}, \mathrm{C}-\mathrm{O}, \mathrm{C}=\mathrm{O}$ and $\mathrm{C}=\mathrm{C}$ bond lengths are shown in Fig. $3 \mathrm{~b}$, whereas complete lists of bond lengths for all calculated structures are available in the ESI $\dagger$ (Table S2). RMSDs for bond lengths are fairly uniform across all the calculations, indicating a consistent description of the covalent molecular structure in full-geometry optimizations regardless of inclusion of dispersion corrections. An average RMSD value of about $0.017 \AA$ is observed for the methods used. This finding is also consistent with our previous calculations performed for the $\mathrm{Li}_{2} \mathrm{C}_{4} \mathrm{O}_{4}{ }^{48}$ and $\mathrm{Li}_{2} \mathrm{DHDMQ}{ }^{6}$ systems. However, the rev-PBE functional diverges from this average value (with a RMSD $=0.022 \AA$ ). The smallest bond length RMSD of $0.011 \AA$ was obtained in the corr-B3PW91-
$\mathrm{D}^{*}{ }_{0} 0.52 / 6-31 \mathrm{G}^{* *}$ dispersion-corrected treatment. PBE-TS, PBEsol and corr-PBE-D*_0.52 methods are characterized by only slightly higher discrepancies with experiment (RMSDs, respectively, are $0.015 \AA 0.015 \AA$ and $0.017 \AA$ ). As a conclusion to the structural features for the TMQ compound, we may consider that the corr-PBE-D*_0.52 and corr-B3PW91-D*_0.52/ $6-31 \mathrm{G}^{* *}$ methods constitute the most suited methodologies for VASP and CRYSTAL calculations respectively in order to account for the geometry of this material (with a better treatment of intermolecular parameters while preserving reasonable accuracy for intramolecular ones). These methodologies will thus be retained for the calculations of the bulk lithiated phase, LiTMQ, corresponding to the reduced form of TMQ. On the other hand, we shall remark that for the TMQ phase at $100 \mathrm{~K}$, the corr-PBE-D*_0.70 treatment results in lattice parameter and unit cell volume discrepancies smaller than $0.5 \%$, whereas the error concerning the inter-plane distance was around $0.9 \%$ (in ESI, $\dagger$ Table S1). Due to the difference in optimal values for the $s_{6}$ parameter according to the temperature, we can deduce that tuning the $s_{6}$ parameter is a way to take into account the temperature effect on the crystal packing interactions, at least from the structural properties viewpoint.

\subsection{Vibrational spectra of TMQ}

Prior to vibrational analysis, the stability and structural features of TMQ molecular and dimeric forms in gas phase are presented. The main spectral features of TMQ will be discussed as obtained from gas-phase DFT calculations of a single molecule and corresponding dimeric forms. Such computational data can indeed be helpful to assess and assign the observed IR bands of TMQ in the crystalline phase. In particular, such studies can elucidate the effect of intermolecular interactions in the crystal phase on experimental vibrational frequencies.

3.2.1 Stability and structural features of TMQ molecular and dimeric forms in gas phase. Isolated molecular TMQ with $C_{\mathrm{i}^{-}}$ symmetry has 78 internal modes of $A_{\mathrm{u}}$ (odd) and $A_{\mathrm{g}}$ (even) symmetry $\left(\Gamma_{\text {vib }}=39 A_{\mathrm{u}}+39 A_{\mathrm{g}}\right.$ ) (see Table S3 in ESI $\dagger$ for calculated structural parameters and Fig. 1c for the schematic structure of the molecule). With this symmetry, the rule of mutual exclusion in IR and Raman spectroscopies holds.

The influence of the interactions taking place between a pair of TMQ molecules on the vibrational bands was also assessed in gas phase. To this end it should be noticed that the structural organization of the TMQ molecules in the unit cell can be described as a tetramer structure (as displayed in Fig. 4). In this figure, two well-defined arrangements between a pair of TMQ molecules can be easily discerned (even if they cannot be disentangled). In the first one, labeled 'dimer 1', the TMQ molecules are found to be nearly in a 'side by side' configuration (Fig. 5). In the second one, the pair of TMQ molecules forms a stacked dimer structure, labeled 'dimer 2' (Fig. 6). The tetramer structure of TMQ (Fig. 4) can thus be considered as a superposition of two pairs of TMQ molecules. These pairs are organized in 'side by side' or in stacked configurations, with statistical weights: $1 / 3$ (dimer 1 ) and $2 / 3$ (dimer 2), respectively (taking into account the symmetry of the crystal cell in the enumeration of pairs). It should be noted 


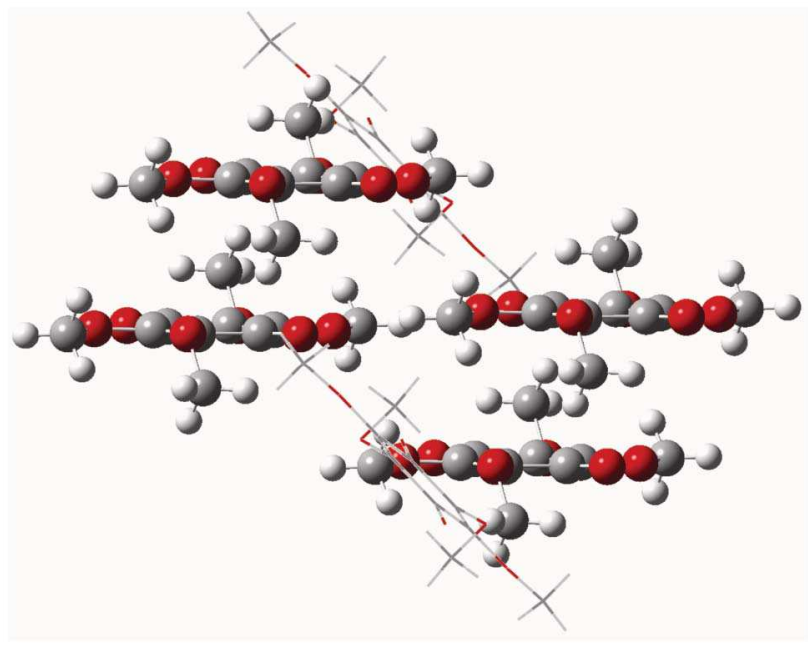

Fig. 4 Ball-and-stick representation of the structural organization of TMQ tetramer in the crystal unit cell along with stick representation of neighboring molecules.

that the main spectral features of TMQ in the condensed phase should originate from induction mechanisms mainly governed by these two types of interactions between the TMQ molecules.

In order to validate this model, we have optimized both dimer structures 1 and 2 in the gas phase. The optimized structural parameters of these dimers are reported in Table S4 of the ESI ${ }^{\dagger}$ and their intermolecular separations are compared with those experimentally determined in crystalline TMQ (Table 1). The dimer structure 1 is found to be more stable (electron energy $\varepsilon_{0}=-1678.96270438 \mathrm{H}$ (or au)) by about 0.8 $10^{-3}$ a.u. (i.e. $2.1 \mathrm{~kJ} \mathrm{~mol}^{-1}$ ) with respect to the dimer structure $2\left(\varepsilon_{0}=-1678.961899 \mathrm{H}\right.$ (or au)). The small difference in energy is equivalent to the thermal bath (at $300 \mathrm{~K}$ ). In dimer structure 1 , the intermolecular distance is evaluated at $8.80 \AA$ (instead of
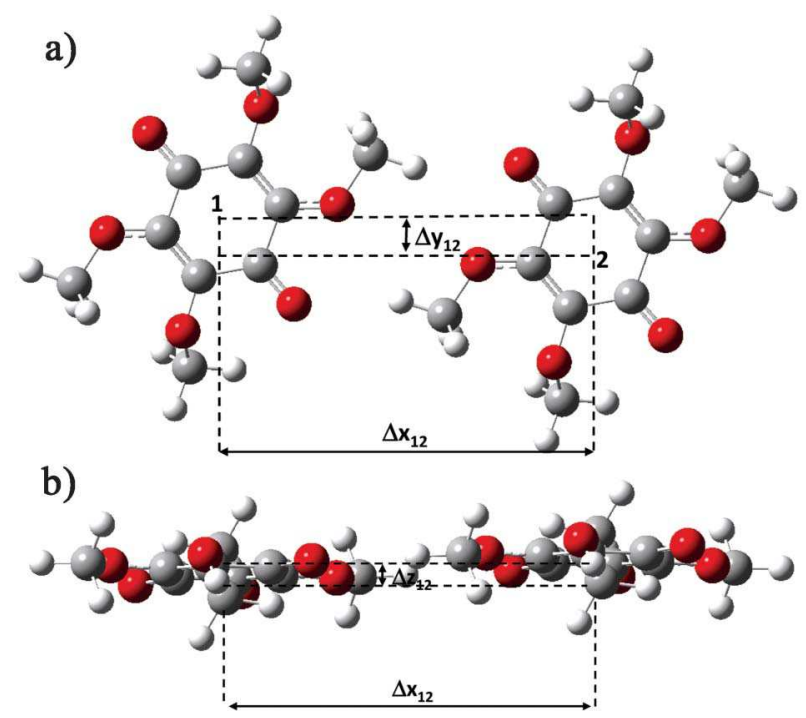

Fig. 5 Representation of the side by side TMQ dimer configuration (dimer 1).
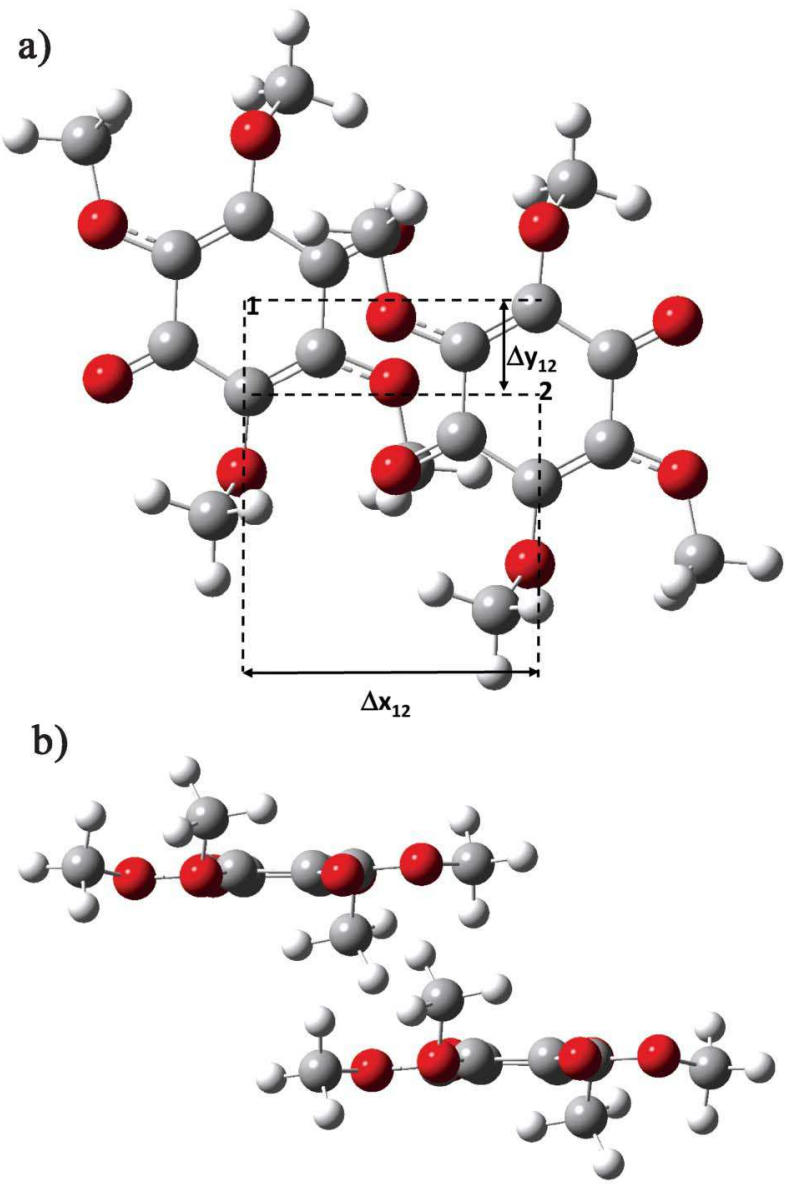

Fig. 6 Representation of the stacked TMQ dimer configuration (dimer 2).

$8.42 \AA$ in the unit cell), whereas the molecular planes are shifted by a separation distance $\Delta z_{12} \sim 0.40 \AA$ (compared to $0.52 \AA$ in the unit cell). In the structure of dimer 2 , the separation distance between TMQ molecules is larger ( $\sim 6.01 \AA$ compared to $4.01 \AA$ in the unit cell), whereas the molecular plane of the partners are shifted by separation distances $\Delta z_{12}\left(\Delta z_{12}=\sqrt{ }\left(\mathrm{Rcm}^{2}-\left(\Delta x_{12}{ }^{2}+\Delta y_{12}{ }^{2}\right)\right)\right.$ and $\Delta \mathrm{y}_{12}$ of $3.80 \AA$ and $0.91 \AA$ respectively (instead of $3.48 \AA$ (calculated inter-plane distance in corr-B3PW91- $\mathrm{D}^{*}{ }_{-} 0.52$ periodic calculation) and $0.68 \AA$ in the crystal phase, respectively). It is well known that the cohesive forces in the crystal lead to a more compact packing arrangement of TMQ dimers than that predicted in a gas phase model, which is particularly observed for the stacked dimer 2. Despite such structural deviations, we shall see in the next section that both gas phase dimer structures reproduce the main spectral features of crystalline TMQ.

3.2.2 Spectral features of TMQ from a gas phase model and comparison with the vibrational spectra in the crystalline state. In this section, we focus on the vibrational analysis of TMQ in gas phase (monomer and dimer structures 1 and 2) in the spectral region $500-3200 \mathrm{~cm}^{-1}$. The assignment of each internal mode (including its wavelength and IR or Raman intensity) of TMQ is provided in Tables S5 and S6 of the ESI. $\dagger$ For the sake of simplicity, in Fig. $7 \mathrm{a}$ and $7 \mathrm{~b}$ we compare the 

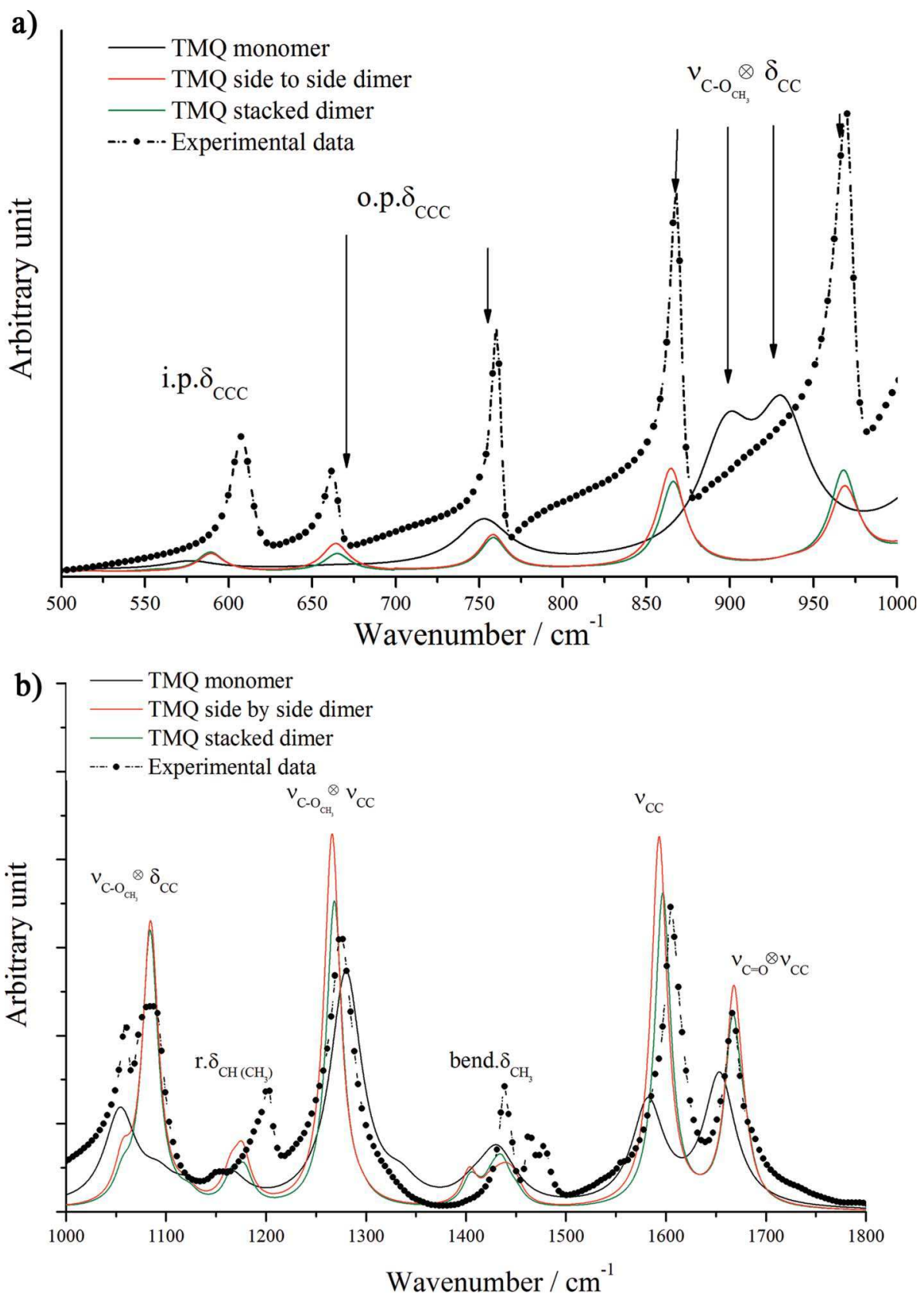

Fig. 7 Comparison of computed vibrational features for the monomer (isolated molecule) and dimeric forms (either stacked or side by side) of TMQ with experimental IR spectra of the TMQ crystal: a) $500-1000 \mathrm{~cm}^{-1}$; b) $1000-1800 \mathrm{~cm}^{-1}$.

calculated IR absorption spectra of monomeric and dimeric forms of TMQ with the experimental spectrum. The calculated frequencies were scaled by a factor of $0.9631 .^{29}$ It has to be noted that the original (i.e. unscaled) frequency values calculated at the B3PW91/6-31+G(2d,p) level are reported in Tables S5 and S6 of the ESI. $\dagger$

We can clearly distinguish different spectral regions associated with specific internal motions of TMQ in either monomeric or dimeric forms. The spectral region 2800-3200 $\mathrm{cm}^{-1}$ is associated with the $\mathrm{C}-\mathrm{H}$ stretching modes of TMQ. From our DFT calculations, the symmetric C-H stretching modes should be located at lower wavenumbers (2915-2940 $\mathrm{cm}^{-1}$ ), whereas the asymmetric $\mathrm{C}-\mathrm{H}$ stretches should be situated towards higher wavenumbers in the $2980-3100 \mathrm{~cm}^{-1}$ spectral region (see uncorrected values in Tables S5 and S6 of the ESI†). Since $\mathrm{C}-\mathrm{H}$ vibrations are significantly anharmonic, couplings between the $\mathrm{C}-\mathrm{H}$ stretching modes and other internal modes of TMQ (having the same symmetry) can induce additional spectral features due to Fermi resonances as well as combination bands. Such couplings between internal modes cannot be disentangled and lead to spectral features in the region of the $\mathrm{C}-\mathrm{H}$ stretching modes which are much more complicated than those predicted from the harmonic vibrational analysis carried out here. Hence, the deviation between 
the calculated and observed spectra in the $\mathrm{C}-\mathrm{H}$ stretching region (figure not shown) suggests that such couplings are present in crystalline TMQ (as well as in the gas phase). This prevents us from proceeding further in the comparative discussion of the spectral signatures of the $\mathrm{C}-\mathrm{H}$ modes of TMQ calculated in the gas phase with those observed in the crystal phase. However, such an analysis remains adequate for the other vibrational modes of TMQ situated in the 500-1800 $\mathrm{cm}^{-1}$ spectral region, which are commented hereafter.

The measured IR bands of TMQ in the crystal phase located at 1680 and $1600 \mathrm{~cm}^{-1}$ are unambiguously assigned to the stretching mode $v_{\mathrm{C}=\mathrm{O}}$ and to the ring stretching mode $v_{\mathrm{C}-\mathrm{C}}$ of TMQ, respectively (Fig. 7). These transitions are also coupled between themselves. There are clear differences between experimental and theoretical results. For example, a shift in the $v_{\mathrm{C}=\mathrm{O}}$ stretching modes of carbonyl groups is observed for both the monomer and dimers of TMQ (the predicted stretch is calculated at about 1650 and $1660 \mathrm{~cm}^{-1}$ for monomer and dimer forms of TMQ, respectively). Similarly, $v_{\mathrm{C}-\mathrm{C}}$ modes are slightly increased by about $10 \mathrm{~cm}^{-1}$ in both dimer structures (1 and 2) with respect to TMQ in gas phase. As a result of dimer formation, these two modes are thus significantly redistributed towards higher vibrational numbers compared with isolated TMQ and are separated by $75 \mathrm{~cm}^{-1}$ (instead of 65 $\mathrm{cm}^{-1}$ in crystal phase). Additionally, their IR intensity is significantly enhanced as a result of the complex formation. The comparison between the calculated IR profiles of both monomer and dimer forms with the IR measurements of crystalline TMQ (Fig. 7) clearly shows that a better agreement is achieved for the dimer structures in the case of these two stretching vibrations. It should be noted that for these two bands, the experimental IR spectrum is already well-reproduced in terms of the predicted wavelengths as well as IR intensity ratios from a gas phase model of both dimeric structures of TMQ.

The IR bands measured in the $1400-1500 \mathrm{~cm}^{-1}$ spectral range can also be unambiguously assigned. In this region, spectral features arise due to both symmetric and asymmetric bending deformations of the methyl groups, $\delta_{\mathrm{C}-\mathrm{H}}\left(\mathrm{CH}_{3}\right)$. It is noteworthy that the asymmetric modes, $\delta_{\mathrm{CH}}\left(\mathrm{CH}_{3}\right)$, are located at slightly higher wavenumbers even though they overlap with the symmetric ones in a limited spectral range. Compared with the isolated TMQ, the interactions between TMQ molecules lead to the formation of a doublet structure in the IR profile, which is also observed in the experimental spectrum.

The intense IR band observed at $1280 \mathrm{~cm}^{-1}$ originates from the coupled $\mathrm{C}-\mathrm{O}$ (methoxy group) and $\mathrm{C}-\mathrm{C}$ stretching modes: $\mathrm{i}$. e. $v_{\mathrm{C}-\mathrm{C}} \otimes v_{\mathrm{C}-\mathrm{O}}$. In the calculated spectra, this band is evaluated at $1273 \mathrm{~cm}^{-1}$ and $1270 \mathrm{~cm}^{-1}$ for the monomer and dimer structures, respectively. It has to be noted that the induced interactions in dimer structures of TMQ play a significant role in the IR intensity enhancement of these modes. The spectral features linked to the $\delta_{\mathrm{CH}}\left(\mathrm{CH}_{3}\right)$ rocking modes of TMQ are located in the spectral region of $1160-1170 \mathrm{~cm}^{-1}$.

At lower wavenumbers $\left(<1100 \mathrm{~cm}^{-1}\right)$, the deviations obtained from the predicted spectra of the monomer and dimer forms of TMQ become more significant (Fig. 7). In the dimer structures, the calculated IR bands located at about 1085, 1105 and $898 \mathrm{~cm}^{-1}$ are associated with the $v_{\mathrm{C}-\mathrm{O}}$ stretching modes of methoxy-groups mixed with the ring deformations, $\delta_{\mathrm{C}-\mathrm{C}}$ of TMQ. The same modes exhibit a doublet structure in the predicted IR profiles of monomer TMQ located at 935 and $900 \mathrm{~cm}^{-1}$, respectively. The bending ring deformation coupled with the methoxy group stretching $\delta_{\mathrm{C}-\mathrm{C}} \otimes v_{\mathrm{C}-\mathrm{O}}$ modes are involved in the splitting of IR bands observed in the $850-1100 \mathrm{~cm}^{-1}$ spectral region. However, the latter is not observed in experiment. It has to be noted that such modes are very sensitive to packing intermolecular interactions occuring in the crystalline TMQ. The other main components of the ring deformation $\delta_{\mathrm{C}-\mathrm{C}}$ modes of in-plane (i.p.) and out-of-plane (o.p.) ring deformations are equally distributed in a broader spectral region ranging from $500-800 \mathrm{~cm}^{-1}$. The o.p. ring deformation $\delta_{\mathrm{C}-\mathrm{C}}$ modes are located at $760 \mathrm{~cm}^{-1}$ for both the monomer and dimer structures, whereas the IR bands calculated at 584 and $662 \mathrm{~cm}^{-1}$ appear to be particularly induced by intermolecular interactions in the dimer structures of TMQ. Despite some deviations expected from the gas phase model, the predicted spectral features obtained from both dimer structures are in fairly good agreement with the observed ones in the solid phase. This finding suggests that the gas-phase dimer structures include an induction mechanism quite similar to that transpired in the vibrational spectra of TMQ in crystal. It can be argued that such a mechanism is mainly governed by the intermolecular packing forces between TMQ molecules.

As a whole, a good agreement is achieved between the theoretical spectral features obtained from a gas phase model of TMQ (dimers) and those obtained from a periodic model of the crystalline TMQ system (Fig. 8). Qualitatively similar trends were observed in the predicted spectral features using both models with the selected DFT procedure. This finding reinforces our previous conclusion about the role of the interaction induced mechanisms in the genesis of the IR profile of TMQ. Tentative mode assignments below $500 \mathrm{~cm}^{-1}$ are out of the scope of this work. However, it is well known that in this spectral region the effects induced by intermolecular interactions on the vibrational spectra are likely to be more pronounced because the vibrational modes exhibit a significant anharmonic character.

\subsection{Electrochemical measurements and intercalation potential prediction}

The electrochemical behavior of TMQ vs. Li measured in a carbonate-based electrolyte was briefly described previously. ${ }^{6}$ Since the aim is to compare the predicted equilibrium potential of the TMQ/LiTMQ system obtained from DFT calculations with experimental values, GITT measurements involving open circuit voltage (OCV) periods were performed to obtain a discharge/charge curve closer to the thermodynamic equilibrium. In agreement with previous data recorded in galvanostatic mode,${ }^{6}$ the shape of the GITT trace confirms the occurrence of a reversible electrochemical reaction with phase transition (weakly polarized) and involving a one-electron process per $\mathrm{C}_{6} \mathrm{O}_{6}$ ring for a potential window ranging from 3.5 to $2.2 \mathrm{~V} v$ s. $\mathrm{Li}^{+} / \mathrm{Li}^{0}$ (Fig. 9). Finally, from relaxation periods, an average OCV value of $2.83 \mathrm{~V}$ was extracted.

From the theoretical point of view, Li intercalation voltage can only be reliably predicted after identifying a thermo- 


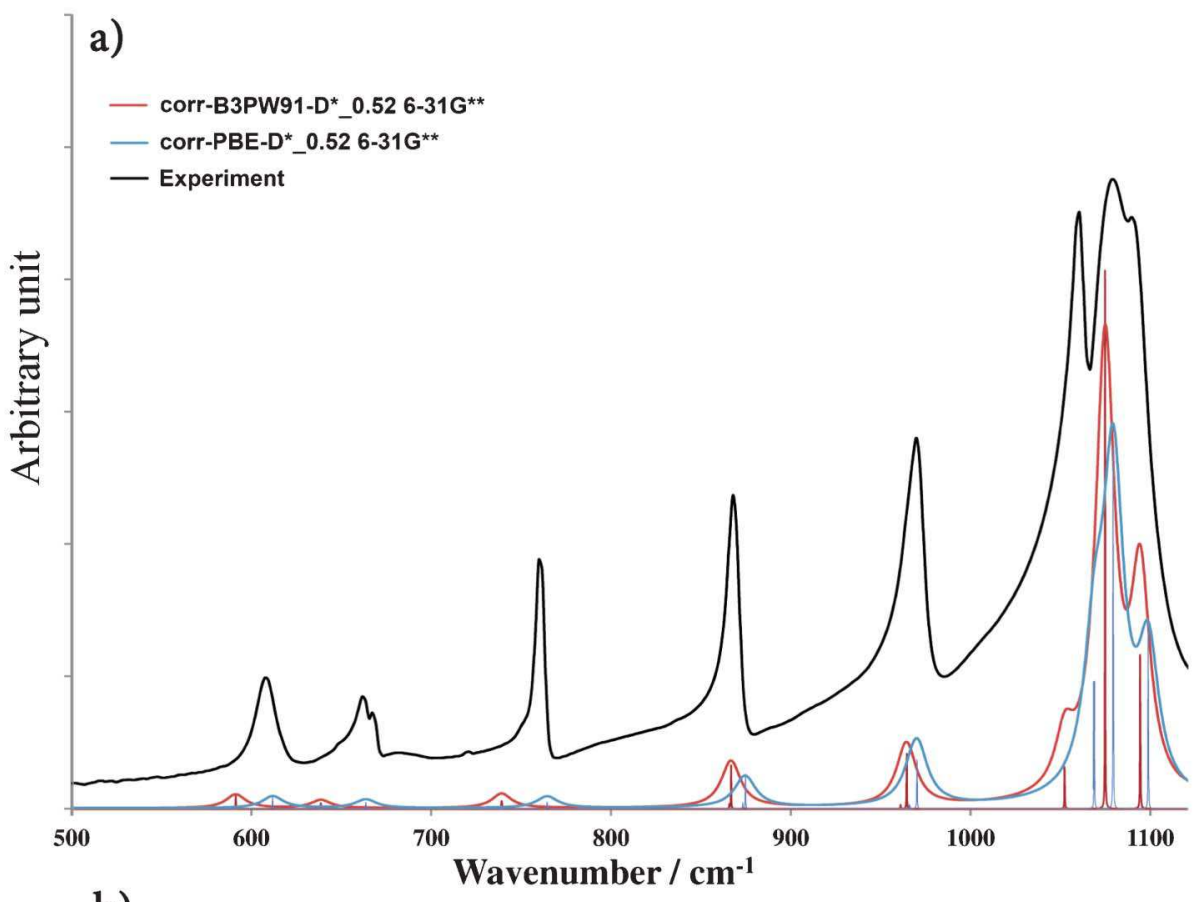

b)

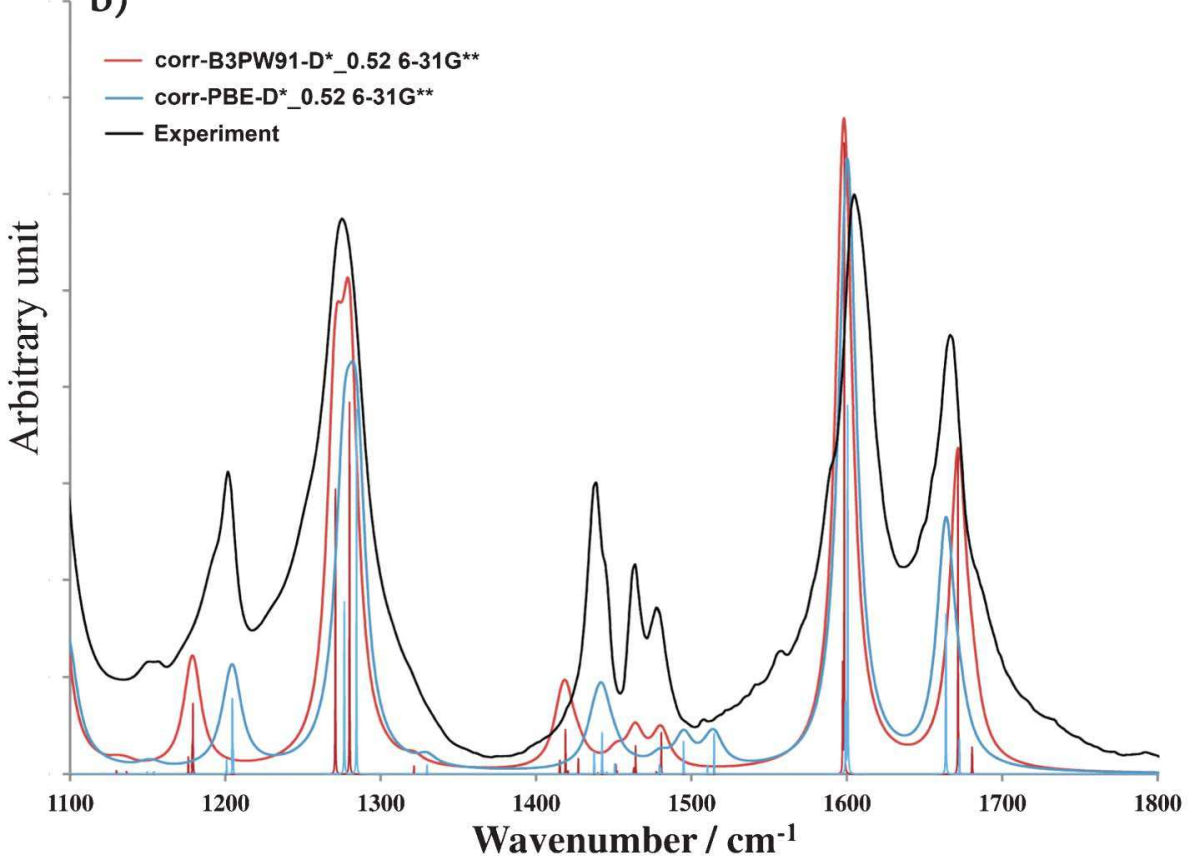

Fig. 8 Simulated and experimental IR spectra of the TMQ crystal: (a) $500-1100 \mathrm{~cm}^{-1}$; (b) $1100-1800 \mathrm{~cm}^{-1}$.

dynamically preferable structure for the lithiated phase (LiTMQ), which is unknown experimentally. Therefore, the first step of such a study should be the search for a possible LiTMQ crystalline structure. Four ways of Li intercalation within TMQ (A, B, C and D models, see Fig. 10) were considered in order to propose a crystal model for the lithiated phase (LiTMQ). Initial positions for the two lithium atoms, which were introduced in the TMQ unit cell, are detailed in ESI. They can be classified as 'aligned' (A), 'right' (B), 'inter- layer' (C), and finally as 'left' (D) with respect to the carbonyl group positioning.

Geometry optimizations of the four hypothetical models for the LiTMQ structure lead to two different final crystal phases, depicted in Fig. 11 and in ESI, $†$ Fig. S1. Among these two final crystal structures, the less stable one (in ESI, $\dagger$ Fig. S1) corresponds to that of crystal B (labeled 'right') with a triclinic cell, which exhibits a difference in energy of $1.12 \mathrm{eV}$ p.f.u. with respect to the more stable one described below. The models A, $\mathrm{C}$ and D as starting structures lead to nearly the same crystal 


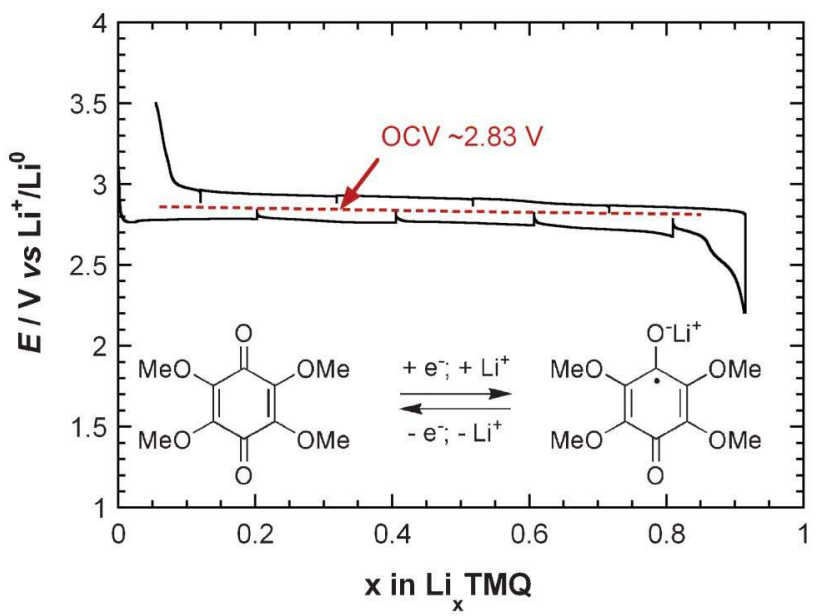

Fig. 9 Typical potential-composition curve for a Li half-cell using TMQ as positive electrode cycled in GITT mode at a rate of one $\mathrm{Li}^{+}$exchanged in $10 \mathrm{~h}$ for $\Delta x=0.1$ followed by a relaxation period of $1 \mathrm{~h}$ (electrolyte: EC/DMC : LiPF $1 \mathrm{M}$ ). The dashed line corresponds to the charge/discharge near-equilibrium potential curve giving an OCV value of $2.83 \mathrm{~V}$. Inset: expected electrochemical insertion/ deinsertion process in TMQ.

after full relaxation, with negligible distinctions in both energy and lattice parameters. The resulting LiTMQ structure determined using the corr-PBE-D*_0.52 treatment in VASP calculations retains the monoclinic unit cell $\left(P 2_{1} / n\right.$ space group, $Z=2$ ) with the following parameters: $a=3.8413 \AA, b=$ $6.8530 \AA, c=19.1766 \AA, \mathrm{V}=524.69 \AA^{3}, \beta=88.710^{\circ}$ (Fig. 11, in ESI, $\dagger$ Table S7). Applying the corr-B3PW91-D*_0.52/6-31G** methodology led to very similar intermolecular geometries: $a=$ $3.7542 \AA, b=6.7768 \AA, c=19.2285 \AA, \mathrm{V}=488.56 \AA^{3}, \beta=87.054^{\circ}$. In this structure, molecules are kept centrosymmetric and lithium atoms lie in the $2 \mathrm{a}$ Wyckoff position. Considering this most stable phase among the hypothetical models tested, the introduction of $\mathrm{Li}$ atoms within the cell induces noticeable changes in the structure. Some gliding effects do occur for the quinonic molecules along the c-axis, leaving one lithium atom in the $(1 / 2,1 / 2,1 / 2)$ position. Considering the van der Waals radius of $\mathrm{Li}$, lithium cation seems to be located at first sight in a distorted octahedral coordination $\left(d_{\max }=2.91 \AA ; d_{\min }=\right.$ $1.90 \AA$ for $\mathrm{Li} \cdots \mathrm{O}$ distances). However, according to our AIM analysis (see section 3.4), the two longest $\mathrm{Li} \cdots \mathrm{O}$ distances $\left(\mathrm{Li} \cdots \mathrm{O}_{2}\right)$, do not correspond to the actual bonding interactions (due to the lack of BCPs). The more realistic polyhedron is thus obtained by retaining only four $\mathrm{Li} \cdots \mathrm{O}$ interactions among the six possible. Therefore, lithium cation lies at the center of a slightly distorted square planar site made of oxygen atoms (Fig. 11). Introduction of lithium induces a tremendous expansion of the $c$ - lattice parameter $(+14.4 \%)$, whereas contraction of $\sim-5 \%$ and $\sim-11 \%$ are respectively observed for the $a$ - and $b$ - axes. Due to such anisotropic but
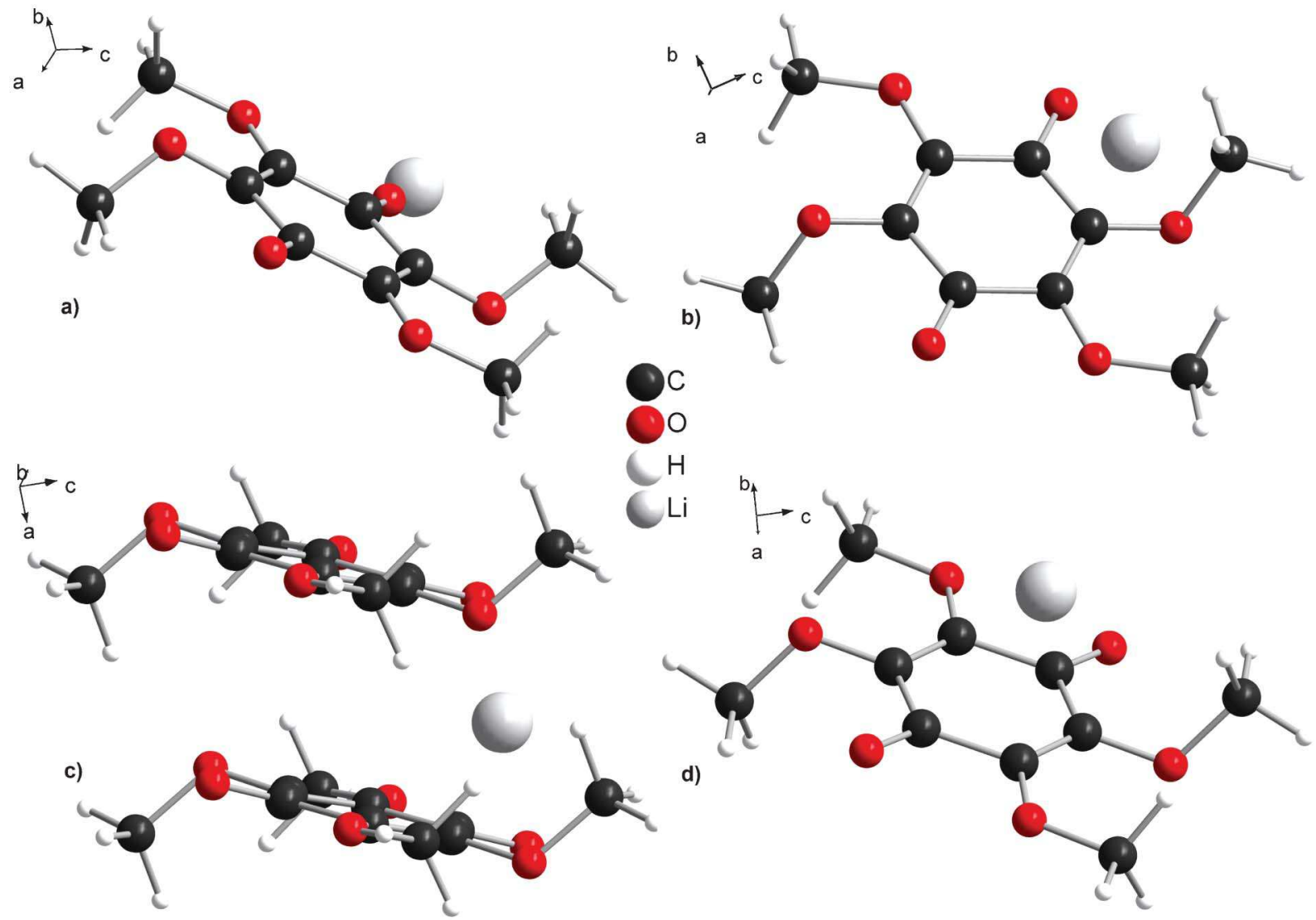

Fig. 10 Representation of Li positioning within the TMQ crystal before relaxation and corresponding labeling: (a) 'aligned' (model A), (b) 'right' (model B) (c) 'interlayer' (model C) (d) 'left' (model D). 


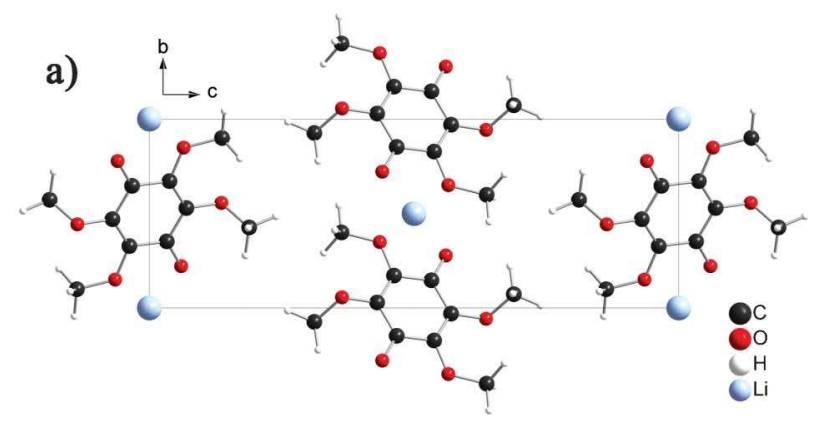

b)

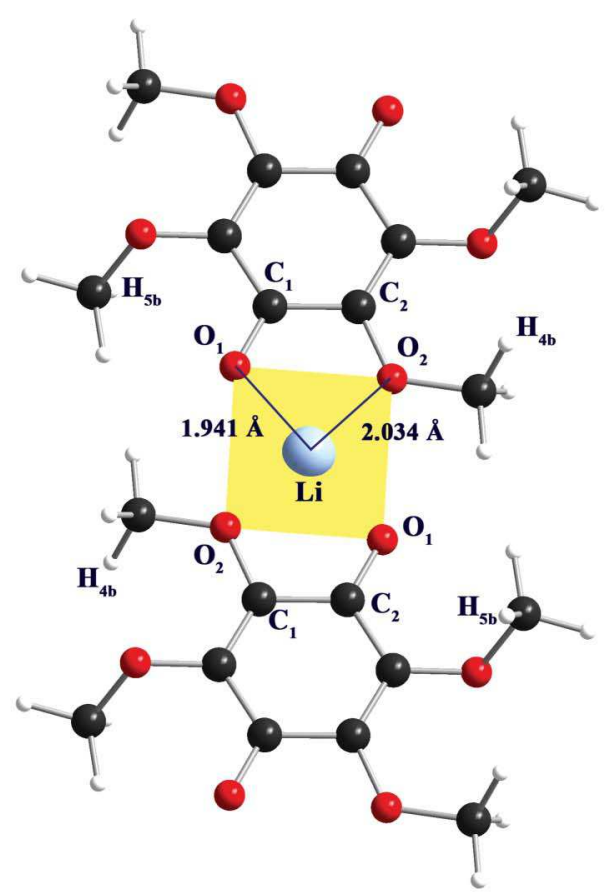

Fig. 11 Predicted crystal structure of the most stable relaxed model for the LiTMQ crystal. (a) View along the a axis; (b) view showing the $\mathrm{LiO}_{4}$ polyhedron.

compensating effects in the structural features, the unit cell volume exhibits a moderate decrease (by $-2.5 \%$ ), which should ensure a reasonable tolerance to the repeated cycling of this material within LiBs in the absence of any side reactions. In this computationally defined LiTMQ phase, $\pi$-stacking is maintained between the radical anions instead of the neutral molecule (Fig. S2b, ESI $\dagger$ ). The ring centroid separation distances of the successive $p$-semiquinone species is lowered of about $0.2 \AA$ compared to the quinonic stacking: i.e. centre-to-centre distance (=length $a$-axis) $\sim 3.8 \AA$ instead of $\sim 4.0 \AA$ for the unlithiated counterpart. On the other hand, intramolecular bonds are found to be affected by the introduction of $\mathrm{Li}$ within the TMQ structure $\left(\Delta d / d\left(\mathrm{C}_{1}-\mathrm{O}_{1}\right)=\right.$ $\left.+3.5 \% ; \Delta d / d\left(\mathrm{C}_{2}-\mathrm{O}_{2}\right)=+3.1 \% ; \Delta d / d\left(\mathrm{C}_{1}-\mathrm{C}_{2}\right)=-3.8 \%\right)$ whereas, as expected, the extent of modifications is much larger for intermolecular ones (in ESI, $\uparrow$ Table S8). This may arise due to the fact that some intermolecular interactions may either appear or disappear in the LiTMQ phase as compared to the unlithiated phase, which will be highlighted through the topological analysis approach (section 3.4). Compared to other solvent-free crystal structures of the alkali salts of semiquinone, the inter-plane distance ( $\mathrm{d}=3.14 \AA$. $3.04 \AA$ and $3.24 \AA$ in the corr-PBE-D*_0.52, corr-B3PW91-D*_0.52 and PBE-TS treatments, respectively) is lower than those observed in $\mathrm{RbBr}_{4} \mathrm{Q}$. $(3.56 \AA)$ and $\mathrm{NaBr}_{4} \mathrm{Q}$. (3.33 $\left.\AA\right) .{ }^{50,51}$ The offset of the molecules is either larger or lower in the LiTMQ structure (approximately equal to $2.2 \AA$ for the best DFT combination, except for PBE-TS, for which the difference was found to be $\sim 2.1 \AA$ ) as compared to the values of $1.54 \AA$ and $2.47 \AA$ for $\mathrm{NaBr}_{4} \mathrm{Q}$ and $\mathrm{RbBr}_{4} \mathrm{Q}$, respectively.

In order to check the stability of the optimized intercalated crystal structure (LiTMQ derivative), i.e. that it actually corresponds to a real minimum on the potential energy surface, we calculated the phonon frequencies at the $\Gamma$ point of the Brillouin zone with the VASP 5.2 package. No unstable modes were found for LiTMQ, therefore demonstrating that no particular structural instability could be related to this phase (i.e. this phase does not correspond to a saddle point along the energy hypersurface).

The voltage for lithium insertion in the TMQ system was evaluated for the more stable structure of LiTMQ. The average intercalation potential of $\mathrm{Li}$ into a host material $v s . \mathrm{Li} / \mathrm{Li}^{+}$can be estimated from DFT calculations through the evaluation of total energies of both lithiated and delithiated phases as well as bulk base centered cubic (bcc) Li. ${ }^{52}$ The average intercalation potential, $\langle V\rangle$, when lithiating a material $\mathrm{Li}_{x} \mathrm{X}$ from $x=$ $x_{1}$ to $x_{2}$, is obtained from eqn (1):

$$
<V>=\frac{-\left[E\left(L i_{x_{2}} X\right)-E\left(L i_{x_{1}} X\right)-\left(x_{2}-x_{1}\right) E(L i)\right]}{\left(x_{2}-x_{1}\right) e}
$$

where $E$ is the total energy as calculated using DFT and $e$ is the absolute value of the electron charge. Since experiments have proven that one lithium ion per formula unit (p.f.u.) can be inserted within TMQ, the overall reaction of the cell can be written as follows:

$$
\mathrm{TMQ}+\mathrm{Li} \rightarrow \mathrm{LiTMQ}
$$

$x_{1}=0$ and $x_{2}=1$ are thus taken as composition limits in the eqn (1). Therefore, the average lithium insertion potential can be extracted from the following expression by considering complete and topotactic lithiation (according to the result for the LiTMQ structure detailed in the previous section):

$$
<V>=E(\mathrm{TMQ})-E(\mathrm{LiTMQ})+E(\mathrm{Li})
$$

As already stated in our previous work dealing with the electrochemical properties of dilithium squarate, ${ }^{48}$ inclusion of the dispersion treatment for $\mathrm{Li}$ bcc metal leads to incorrect lattice parameter values resulting from an overestimation of these long range forces. Therefore, average intercalation voltage presented below corresponds to calculations with the PBE treatment of bcc $\mathrm{Li}$ (which are more physically reasonable). Using the corr-PBE-D*_0.52 treatment for both the TMQ and LiTMQ phases, a perfect agreement with the GITT measurement is found for the intercalation voltage, with a discrepancy lower than $1 \%$ with respect to the experiment (i.e. 2.84 V vs. $2.83 \mathrm{~V}$ ). 


\subsection{Bond critical points, atomic charges and $\pi$-electron delocalization analysis of the TMQ and LiTMQ systems}

Topological features ${ }^{23}$ can be used to complement the abovementioned theoretical results by providing some quantitative descriptors of the chemical bonding. Such data were collected for the TMQ and LiTMQ phases. A BCP corresponding to zero gradient of electron density is found between each pair of nuclei, which are considered to be linked by a chemical bond with two negative curvatures $\left(\lambda_{1}\right.$ and $\left.\lambda_{2}\right)$ and one positive curvature $\left(\lambda_{3}\right)$, denoted as a $(3,-1)$ critical point. The Laplacian value is a measure of the local curvature of $\rho(\mathrm{r})$ and indicates areas that correspond to either excess of the electron density $\left(\nabla^{2} \rho(\mathrm{r})<0\right)$ or depletion of the electron density $\left(\nabla^{2} \rho(\mathrm{r})>0\right)$ at a given point in space. The bond ellipticity, $\varepsilon$, defined in terms of the two negative curvatures $\lambda_{1}$ and $\lambda_{2}$ as $\varepsilon=\left(\lambda_{1} / \lambda_{2}-1\right)$, shows the deviation of the charge distribution of a bond path from axial (cylindrical) symmetry. The ellipticity at the BCP can thus be interpreted as a measure of the anisotropy of the curvature of the electron density in the directions perpendicular to the bond (a zero value indicating no anisotropy), and therefore serves as a sensitive index to monitor the $\pi$-character of double bonds. Kinetic energy density, $G$ - which is an always positive value related to the ionic character ${ }^{53}$ - can be used to classify bonds according to the extent of its ratio to electron density, $G / \rho_{\mathrm{BCP}}$. A value of $G /$ $\rho_{\mathrm{BCP}}>1.0$ is found for closed shell systems, whereas values $<$ 1.0 are indicative of shared interactions. ${ }^{23}$ From the potential energy density, $V$ - that is always negative - a measure of the covalency $^{23}$ or the hydrogen bond strength through various empirical relationships ${ }^{54,55}$ can be determined. Additionally, the total energy density, $H$, the sum of $G$ and $V$, also allows for a classification of bonds, with positive values being associated with closed shell interactions and negative ones with shared interactions. $^{23}$ The mentioned quantities for the various interactions occurring in the TMQ/LiTMQ crystals are presented in Tables S9-S12 of the ESI. $\dagger$ For the sake of comparison, BCPs of corresponding TMQ molecule and $\mathrm{Li}^{+}$ $\mathrm{TMQ}^{-}$complex are provided in Table S13, ESI, $\dagger$ along with Fig. S3, ESI, $\dagger$ showing positions of BCPs or ring critical points (RCPs) on these molecular systems.

The topological parameters for the $(3,-1)$ BCPs on the covalent bonds for TMQ/LiTMQ crystals are listed in Tables S9 and S10 of the ESI. $\dagger$ All covalent bonds of the TMQ and LiTMQ compounds (except the covalent/polar $\mathrm{C}_{1}-\mathrm{O}_{1}$ one in the TMQ phase) are characterized by negative values of the Laplacian of $\rho$ at the BCPs. In the case of the $\mathrm{C}_{1}-\mathrm{O}_{1}$ bond, the BCP is so close to the $\mathrm{C}$ atom that it falls in the core region of charge depletion of the $\mathrm{C}$ atom, generating a positive value for $\nabla^{2} \rho$ (i.e., the valence shell of $\mathrm{C}$ is transferred in the $\mathrm{O}$ atom basin and is no longer visible in the $\mathrm{C}$ basin). This effect is still observed for molecular TMQ (Table S13 and Fig. S3b, ESI $\dagger$ ). For the $\mathrm{C}-\mathrm{C}$ and $\mathrm{C}-\mathrm{O}$ type bonds, the values of the total density and its Laplacian at the BCP are in good agreement with the results obtained for the previously studied quinonic system, $\mathrm{Li}_{2}$ DHDMQ. ${ }^{6}$ For the homonuclear $\mathrm{C}-\mathrm{C}$ bonds, the BCP is approximately centered between the two $\mathrm{C}$ atoms. In contrast, for the heteronuclear $\mathrm{C}-\mathrm{O}$ bonds, the BCP lies significantly closer to the $\mathrm{C}$ atom, in accordance with a greater accumula- tion of density concentration in the more electronegative $\mathrm{O}$ atom.

In both TMQ and LiTMQ crystals, the C-C bonds exhibit values of $\rho$ and absolute values of $\nabla^{2} \rho$ that systematically decrease with increasing bond length, which corresponds to increasing single bond character. This differentiation in the $\mathrm{C}-\mathrm{C}$ bond lengths within the ring might arise due to the intermolecular interactions. To that respect, it should be mentioned that the bond lengths observed in experiment correlated well with the predicted ones, which further demonstrates the ability of the theoretical treatment to predict properties of real systems. This observation is corroborated by the value of $\varepsilon$, which clearly increases with increasing $\pi$ character in these two forms (TMQ and LiTMQ) as well. In the TMQ phase, the extent of $\rho(\mathrm{r})$ at BCP for the $\mathrm{C}-\mathrm{O}$ bond also systematically decreases with increasing bond length. Among all the $\mathrm{C}-\mathrm{O}$ bonds, $\mathrm{C}_{1}-\mathrm{O}_{1}$ appears to be the strongest in terms of its $\rho\left(r_{\mathrm{C}}\right)$ value, showing its double-bond character. For the LiTMQ phase, a similar trend is found, with a slightly higher extent of $\rho(\mathrm{r})$ at BCP being observed for $\mathrm{C}_{3}-\mathrm{O}_{3}$ as compared to $\mathrm{C}_{2}-\mathrm{O}_{2}$. The $\mathrm{C}_{4}-\mathrm{O}_{2}$ and $\mathrm{C}_{5}-\mathrm{O}_{3}$ show similar results. On the other hand, $\mathrm{C}-\mathrm{H}$ bonds, all belonging to the methyl groups, are characterized by very similar topological features.

The molecules in the TMQ crystal lattice display strong (covalent) bonds and are held together through weaker intermolecular interactions. The details of all the parameters characterizing such interactions are given in Tables S11 and S12 of the ESI. $\dagger$ All of these interactions in the two forms are characterized by a $\rho$ value $<1$, a positive Laplacian, and a ratio of $\lambda_{1} / \lambda_{3}<1$. As expected, the interactions in TMQ can therefore be classified as closed shell interactions. As already noticed for other closed shell interactions, ${ }^{56}$ all these interactions are characterized by a ratio $V / G<1$ (in ESI, † Table S12). The TMQ compound generates various intermolecular interactions such as $\mathrm{C} \cdots \mathrm{O}-\mathrm{C}, \mathrm{C} \cdots \mathrm{H}-\mathrm{C}, \mathrm{O} \cdots \mathrm{H}-\mathrm{C}$, and $\mathrm{H} \cdots \mathrm{H}-\mathrm{C}$ interactions. The criterion ${ }^{57}$ of $\rho(\mathrm{r})$ at BCP within 0.0130.236 e $\AA^{-3}$ for a hydrogen bond is predicted for all $\mathrm{O} \cdots \mathrm{H}-\mathrm{C}$ interactions, which are similar to those observed for $\mathrm{O} \cdots \mathrm{H}-$ $\mathrm{C}^{\text {methyl }}$ interactions in the $\mathrm{Li}_{2} \mathrm{DHDMQ}^{6}$ systems. $\mathrm{H} \cdots \mathrm{H}-\mathrm{C}$ interactions, which were absent from $\mathrm{Li}_{2} \mathrm{DHDMQ},{ }^{6}$ also fall within the same range of magnitude of $\rho(\mathrm{r})$ characteristic for hydrogen bonds.

For the LiTMQ compound, additional O $\cdots \mathrm{Li}$ interactions are observed. In terms of the total energy density, the $\mathrm{O} \cdots \mathrm{Li}$ interactions are found to be the strongest (in agreement with interaction distances, $R_{\mathrm{A}-\mathrm{B}}$ ) followed by $\mathrm{C} \cdots \mathrm{O}-\mathrm{C}$ and $\mathrm{C} \cdot{ }^{\cdots} \mathrm{H}-\mathrm{C}$ (in ESI, $†$ Table S10). Although fewer in number than observed for the $\mathrm{Li}_{2} \mathrm{DHDMQ}^{6}$ phases, the $\rho(\mathrm{r})$ and $\nabla^{2} \rho$ values at BCPs for the LiTMQ Li $\cdots \mathrm{O}$ interactions are larger due to the higher strength of these interactions, which is reflected by shorter $\mathrm{Li} \cdots \mathrm{O}$ distances than those observed in the $\mathrm{Li}_{2} \mathrm{DHDMQ}^{6}$ compounds. Additionally, some additional $\mathrm{C} \cdots \mathrm{H}-\mathrm{C}$ and $\mathrm{H} \cdots \mathrm{H}-\mathrm{C}$ interactions occur, whereas some (and sometimes many) of the $\mathrm{O} \cdots \mathrm{H}-\mathrm{C}$ and $\mathrm{H} \cdots \mathrm{H}-\mathrm{C}$ interactions are no longer present as compared with the TMQ compound. In particular, due to the combined molecular gliding effect and lithium ion positioning in between the $\mathrm{O}_{1}$ and $\mathrm{O}_{2}$ atoms, $\mathrm{O}_{1} \cdots \mathrm{H}-\mathrm{C}$ and $\mathrm{O}_{2} \cdots \mathrm{H}-\mathrm{C}$ hydrogen bonds disappear upon lithium intercalation. Only those involving the $\mathrm{O}_{3}$ atom are maintained. The 


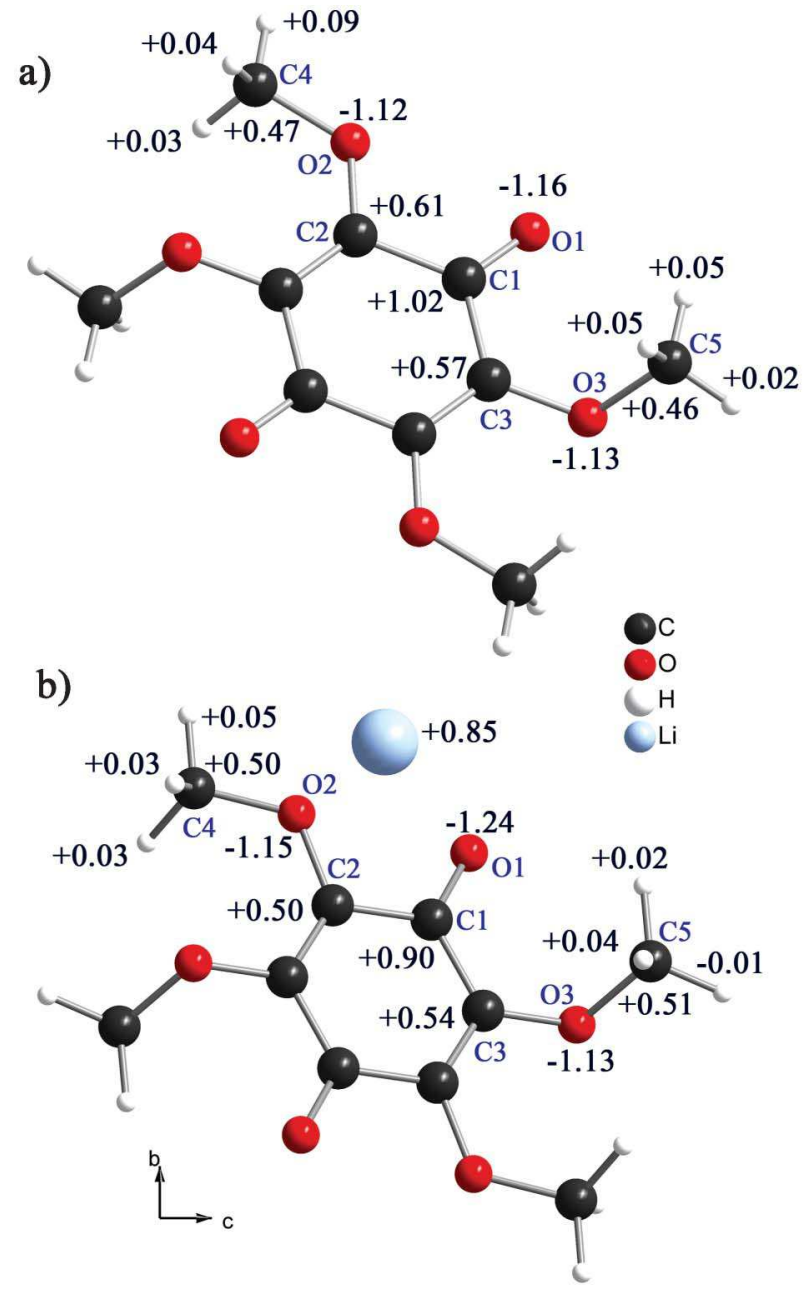

Fig. 12 Net atomic charges for calculated structures: (a) in the TMQ crystal (b) in the most stable form of LiTMQ crystal.

intramolecular $\mathrm{C}_{4} \cdots \mathrm{O}_{3}-\mathrm{C}_{3}$ interaction is of slightly higher strength in the lithiated phase compared to the TMQ form due to smaller interatomic distances, generating higher values of $\rho(\mathrm{r})$ and $\nabla^{2} \rho$ at the BCP. Similarly, a higher strength for $\mathrm{C}_{1} \cdots \mathrm{H}_{4 \mathrm{c}}-\mathrm{C}_{4}$ is found for LiTMQ as compared with TMQ. In addition, intramolecular $\mathrm{C}_{1} \cdots \mathrm{H}_{5 \mathrm{c}}-\mathrm{C}_{5}$ and intermolecular $\mathrm{C}_{1} \cdots \mathrm{H}_{5 \mathrm{c}}-\mathrm{C}_{5}$ interactions are generated as a result of lithium insertion in the TMQ matrix.

The net charges of atomic species for TMQ and LiTMQ crystals are reported in Fig. 12. Due to the polar bonding character, net atomic charges (NACs) in the TMQ molecule are far from the formal oxidation states. As already observed for the dilithium squarate crystal, $^{48}$ the NAC of lithium in the LiTMQ crystal is around $+0.9\left(\mathrm{q}_{\mathrm{Li}}=+0.85\right)$, indicating an almost full one-electron transfer from $\mathrm{Li}$ to the adjacent TMQ molecule. A global redistribution of electron density around each atomic species accompanies this lithiation phenomenon, with, for instance, a global decrease of the oxidation state for hydrogen atoms belonging to the methyl groups, at least partially compensated by slight increase of carbon atom oxidation states on the same functional group. Thus, the reduction phenomenon does not imply solely the carbonyl group redox-active centre $\left(\mathrm{C}_{1}=\mathrm{O}_{1}\right)$. Indeed, only $0.2 \mathrm{e}$ is transferred to this group upon lithium insertion and not 0.85 e. Similarly, a value of nearly $0.42-0.43$ e should be additioned on each carbonyl group for an exclusive transfer partitioned between these two redox centres without affecting other atoms in the bulk crystal structure. In fact, 0.14 e is also shifted to the $\mathrm{C}_{2}-\mathrm{O}_{2}$ bond, which is as near to the $\mathrm{Li}$ as $\mathrm{C}_{1}-\mathrm{O}_{1}$. Even if $\mathrm{O}$ atoms close to $\mathrm{Li}\left(\mathrm{O}_{1}\right.$ and $\left.\mathrm{O}_{2}\right)$ are characterized by greater negative charge after lithium reduction, we shall note that charge transfer (CT) decreases from 1.73 to 1.65 for $\mathrm{C}_{2}-\mathrm{O}_{2}$ and from 2.18 to 2.14 for $\mathrm{C}_{1}-\mathrm{O}_{1}$ upon $\mathrm{Li}$ intercalation. Indeed, such clear indication of increased covalency is confirmed by $|V| / G$ raising, which is characteristic of bond lengthening for $\mathrm{C}-\mathrm{O}$. Other signatures of this phenomenon are seen in $\nabla^{2} \rho$ that becomes negative instead of positive for $\mathrm{C}_{1}-\mathrm{O}_{1}$ and more negative for $\mathrm{C}_{2}-\mathrm{O}_{2}$. Similarly, $\lambda_{3}$ largely decreases upon $\mathrm{Li}$ intercalation. For $\mathrm{C}_{1}-\mathrm{O}_{1}$ such effect might be related to the reduction of double bond character.

As a whole, changes in atomic charges upon Li intercalation significantly modify the energy landscape in the alkali salt of the semiquinone anion radical as compared to the quinone, in particular from the viewpoint of crystal lattice energy, through the Madelung constant or dipole momentum modifications and by adjunction of ion-dipole interactions as well. The incidence of Coulombic interactions/Madelung constant evolution was already noticed upon diffusion within doped ceria matrices ${ }^{58}$ or in link with (de-)intercalation for either organic ${ }^{48}$ or inorganic compounds. ${ }^{38}$ Even if the material under study corresponds initially to a molecular crystal (TMQ) it is transformed into an organic salt after lithiation (LiTMQ) for which electrostatic interactions are prevailing. Explicit Madelung calculations using the Eugen code ${ }^{59}$ were performed to account for much lower stability of the LiTMQ model characterized weaker electrostatic energy (more than $1 \mathrm{eV}$ ) compared to the stable one. Indeed, lattice energies obtained from the Madelung constant prediction are characterized by -450.6 and $-494.2 \mathrm{~kJ} \mathrm{~mol}^{-1}$ for these two LiTMQ phases, respectively.

Finally, the electronic delocalization has been analyzed in both molecular and crystal systems by using either geometry considerations or precise data of electronic structure already taken into account for BCP observations. The following descriptors of $\pi$-electron delocalization have been used: (i) the harmonic oscillator model of aromaticity (HOMA), ${ }^{42,43}$ which is geometry-based index of aromaticity (displayed in Table 2); (ii) two descriptors based on topological properties of the charge density: FLU ${ }^{44,45}$ and $\mathrm{H}$ value at the ring critical point $^{60}$ (see section 1.3 in ESI $\dagger$ for details on the two first descriptors). The HOMA value (Table 2) for ring in molecular TMQ $(-0.75)$ indicates slightly lower $\pi$-electron delocalization extent than the one found for $p$-benzoquinone ( $p$-BQ) $(-0.61) .{ }^{50}$ This may arise due to the longer $\mathrm{C}=\mathrm{C}$ bond $(1.36 \AA)$ in TMQ with respect to $p$-BQ $(1.33 \AA)$, whereas values for $\mathrm{C}-\mathrm{C}$ bonds are identical. Upon lithium reduction of TMQ, the resulting $p$-semiquinone radical exhibits a more delocalized ring, with HOMA value reaching 0.36. FLU indices (available only for the molecular state), confirm these trends, with values equal to 0.130 and 0.036 for TMQ and LiTMQ 
Table 2 HOMA values for ring and specific paths along the species in molecular and crystal TMQ/LiTMQ forms

\begin{tabular}{|c|c|c|c|c|c|}
\hline & TMQ & LiTMQ & TMQ & LiTMQ-stable & LiTMQ-less stable \\
\hline $\mathrm{O}_{2}-\mathrm{C}_{2}-\mathrm{C}_{3}-\mathrm{C}_{1}-\mathrm{O}_{1}$ & 0.170 & 0.523 & 0.113 & 0.291 & 0.322 \\
\hline $\mathrm{O}_{3}-\mathrm{C}_{3}-\mathrm{C}_{2}-\mathrm{C}_{1}-\mathrm{O}_{1}$ & -0.140 & 0.486 & -0.442 & 0.131 & -0.226 \\
\hline $\mathrm{C}_{3}-\mathrm{C}_{2}-\mathrm{C}_{1}-\mathrm{O}_{1}$ & 0.175 & 0.785 & 0.390 & 0.740 & 0.520 \\
\hline $\mathrm{C}_{2}-\mathrm{C}_{3}-\mathrm{C}_{1}-\mathrm{O}_{1}$ & -0.132 & 0.748 & -0.391 & 0.630 & 0.364 \\
\hline
\end{tabular}

compounds, respectively. Indeed, a decrease in FLU values is related to a $\pi$-electron delocalization increase. Such effects are also maintained in the solid state, with similar values of HOMA amounting to -0.88 and 0.31 for TMQ and stable LiTMQ crystal phases, respectively. Similarly, slight enhancement of $\mathrm{H}$ values for RCPs between TMQ (0.007 a.u.) and LiTMQ (0.008 a.u.) crystals also confirm the trend. The slight departure of HOMA for the TMQ crystal as compared to the molecule can be ascribed to a larger average value of CC bond distances in the ring at the solid state. The TMQ crystalline phase is characterized by much less aromaticity features if compared to the ring HOMA values reached by $\mathrm{NaBr}_{4} \mathrm{Q}$ and $\mathrm{RbBr}_{4} \mathrm{Q}$ forms amounting to 0.46 and 0.43 respectively, according to the crystal data reported in. ${ }^{50}$ On the other hand, it is worth noticing that distinct Li positioning occurring for the less stable LiTMQ phase induces less efficient $\pi$-electron delocalization than in the stable counterpart, with HOMA amounting only to 0.06 . In addition to distinct ion-dipole or dipole-dipole,... interactions, this effect may take part to the differentiation in energy of these two phases. In complement to ring features, two other additional delocalization paths were studied $\left(\mathrm{O}_{2}-\mathrm{C}_{2}-\mathrm{C}_{3}-\mathrm{C}_{1}-\mathrm{O}_{1}\right.$ and $\mathrm{O}_{3}-\mathrm{C}_{3}-\mathrm{C}_{2}-\mathrm{C}_{1}-\mathrm{O}_{1}$ according to labeling presented in Fig. S3a, ESI $\dagger$ ) exhibiting enhanced electron sharing for the neutral molecule (TMQ) with respect to the ring, especially for the first one. In the TMQ crystal phase, the trend of delocalization enhancement as compared with the ring is confirmed for these two paths (HOMA $=0.11$ and -0.44 , respectively). For the LiTMQ radical anion, these two paths override the ring values, whereas for most stable LiTMQ crystal corresponding values characterize slightly less $\pi$-electron delocalization than in the ring. In the unstable LiTMQ phase, both paths exhibit noticeable aromaticity contrary to the ring. Finally, two further paths excluding the methoxy oxygen $\left(\mathrm{C}_{3}-\mathrm{C}_{2}-\mathrm{C}_{1}-\mathrm{O}_{1}\right.$ and $\left.\mathrm{C}_{2}-\mathrm{C}_{3}-\mathrm{C}_{1}-\mathrm{O}_{1}\right)$ were found to be characterized by high $\pi$-electron delocalization in LiTMQ (both molecular and stable crystalline forms) systems, with HOMA values comprised between 0.63 and 0.79 .

\section{Conclusion}

In the search for improved battery electrodes, redox organic electrodes might provide a way to reduce both the ecological footprint and restricted resources imposed by the current use of rare metal oxides. In this study, we reported the electrochemical properties of TMQ investigated by using first principles calculations at the DFT level of theory. The voltage is measured to be $\sim 2.8 \mathrm{~V} v s$. $\mathrm{Li} / \mathrm{Li}^{+}$, with a low polarization effect. Our simulation showed good reproduction of the observed crystal structure of TMQ at RT by using either the corr-PBE-D*_0.52 or the corr-B3PW91-D*_0.52/6-31G** methods, therefore rendering pure DFT (GGA-PBE or LDA) as well as PBE-D treatments unreliable. Tuning the $s_{6}$ parameter in Grimme's dispersion correction allows us to account for the lattice parameter changes during temperature cooling.

Computational studies based on DFT methods have also advanced our understanding, at the atomic level, of potential lithium insertion sites in the TMQ battery material. Among several models of intercalation, the most energetically stable one was found to exhibit no indication of structural instability (i.e. due to the lack of imaginary frequencies). The most favorable lithium insertion site for a complete lithiation $(x=1$ for $\mathrm{Li}_{x} \mathrm{TMQ}$ ) is found to be a slightly distorted square planar site. For this phase, a small lattice volume change during the charge/discharge process was found, which should make it a promising cathode material for the rechargeable LiB. Considering a biphasic TMQ/LiTMQ reversible system, the calculated half-cell voltage $(2.84 \mathrm{~V})$ with the corr-PBE-D ${ }^{*} 0.52$ method is perfectly compatible with the near-equilibrium potential curve giving an OCV value of $2.83 \mathrm{~V}$.

The Bader topological analysis related to the distribution of the electron density and the associated parameters in intermolecular space was used as a quantitative measure to compare the strength of the covalent and intermolecular interactions in TMQ and LiTMQ phases. Analysis of $\pi$-electron delocalization descriptors provided useful insights into the aromaticity changes upon lithiation, outlining both similarities in ring features between molecular and crystal state as well as identification of most favored delocalization pathways or incidence of $\mathrm{Li}$ positioning in the unit cell with respect to such effects.

Due to the organic character of the compound, the prediction of its molecular properties can be assessed through using single molecule or larger cluster systems modeling. This study has indeed provided the probe of a very reliable methodology for calculations of the vibrational spectra of crystalline TMQ in the mid-IR range based on DFT calculations of a dimer model. Larger deviations of the theoretical results from experiment were observed for the isolated molecule (monomer form), therefore highlighting an important role of packing forces on the vibrational modes. 


\section{Acknowledgements}

The authors gratefully acknowledge the GCEP sponsors within the GCEP program (http://gcep.stanford.edu/) as well as the region Picardie and the FEDER program for financial support of the project. The authors deeply thank J. Geng for preliminary experiments, J. Mascetti (ISM-CNRS UMR 5255, France) for FT-IR measurements, N. Penin and O. Viraphong (ICMCB-CNRS UPR 9048, France) for ball milling proir to FT-IR measurements as well as F. Dolhem (LG-CNRS FRE 6219, France) for fruitful discussions. We thank the DSI-CCuB from the University of Bourgogne, the computer center MCIA of the University of Bordeaux and Pays de l'Adour and the CINES from Montpellier for allowing us to access their computer facilities. We gratefully acknowledge generous allocations of computing time from the CINES. EII gratefully acknowledges the National Computational Infrastructure Facilities in Canberra for generous allocations of computer time and the Australian Research Council for a DP grant and a Future Fellowship.

\section{References}

1 H. Chen, M. Armand, G. Demailly, F. Dolhem, P. Poizot and J.-M. Tarascon, ChemSusChem, 2008, 1, 348.

2 H. Chen, M. Armand, M. Courty, M. Jiang, C. P. Grey, F. Dolhem, J.-M. Tarascon and P. Poizot, J. Am. Chem. Soc., 2009, 131, 8984.

3 H. Chen, P. Poizot, F. Dolhem, N. I. Basir, O. Mentré and J.M. Tarascon, Electrochem. Solid-State Lett., 2009, 12, A102.

4 M. Armand, S. Grugeon, H. Vezin, S. Laruelle, P. Ribière, P. Poizot and J. M. Tarascon, Nat. Mater., 2009, 8, 120.

5 S. Renault, J. Geng, F. Dolhem and P. Poizot, Chem. Commun., 2011, 47, 2414.

6 A.-L. Barrès, J. Geng, G. Bonnard, S. Renault, S. Gottis, O. Mentré, C. Frayret, F. Dolhem and P. Poizot, Chem.-Eur. J., 2012, 18, 8800.

7 W. Walker, S. Grugeon, O. Mentré, S. Laruelle, J.M. Tarascon and F. Wudl, J. Am. Chem. Soc., 2010, 132, 6517.

8 W. Walker, S. Grugeon, H. Vezin, S. Laruelle, M. Armand, F. Wudl and J.-M. Tarascon, J. Mater. Chem., 2011, 21, 1615.

9 Y. Hanyu and I. Honma, Sci. Rep., 2012, 2.

10 J. Xiang, C. Chang, M. Li, S. Wu, L. Yuan and J. Sun, Cryst. Growth Des., 2008, 8, 280.

11 M. Yao, H. Senoh, M. Araki, T. Sakai and K. Yasuda, ECS Trans., 2010, 28, 3.

12 Y. Morita, S. Nishida, T. Murata, M. Moriguchi, A. Ueda, M. Satoh, K. Arifuku, K. Sato and T. Takui, Nat. Mater., 2011, 10, 947.

13 Z. Song and H. Zhou, Energy Environ. Sci., 2013, 6, 2280.

14 Y. Liang, Z. Tao and J. Chen, Adv. Energy Mater., 2012, 2, 742.

15 P. Poizot and F. Dolhem, Energy Environ. Sci., 2011, 4, 2003.

16 L. J. Sham and W. Kohn, Phys. Rev., 1966, 145, 561.

17 J. P. Perdew, K. Burke and M. Ernzerhof, Phys. Rev. Lett., 1996, 77, 3865.

18 Y. Zhang and W. Yang, Phys. Rev. Lett., 1998, 80, 890.
19 J. P. Perdew, A. Ruzsinszky, G. I. Csonka, O. A. Vydrov, G. E. Scuseria, L. A. Constantin, X. Zhou and K. Burke, Phys. Rev. Lett., 2008, 100, 136406.

20 A. D. Becke, J. Chem. Phys., 1993, 98, 5648.

21 S. Grimme, J. Comput. Chem., 2006, 27, 1787.

22 A. Tkatchenko and M. Scheffler, Phys. Rev. Lett., 2009, 102, 073005.

23 R. F. W. Bader, Atoms in Molecules: A Quantum Theory, Clarendon Press ed. Oxford, 1990.

24 H. S. Verter and J. Rogers, J. Org. Chem., 1966, 31, 987.

25 SADABS: Area-Detector Absorption Correction, 1996.

26 G. M. Sheldrick, SHELXTL, 1998.

27 V. Petricek, M. Dusek and L. Palatinus, JANA2006, 2006.

28 M. J. Frisch, G. W. Trucks, H. B. Schlegel, G. E. Scuseria, M.

A. Robb, J. R. Cheeseman, G. Scalmani, V. Barone, B. Mennucci, G. A. Petersson, H. Nakatsuji, M. Caricato, X. Li, H. P. Hratchian, A. F. Izmaylov, J. Bloino, G. Zheng, J. L. Sonnenberg, M. Hada, M. Ehara, K. Toyota, R. Fukuda, J. Hasegawa, M. Ishida, T. Nakajima, Y. Honda, O. Kitao, H. Nakai, T. Vreven, J. J. A. Montgomery, J. E. Peralta, F. Ogliaro, M. Bearpark, J. J. Heyd, E. Brothers, K. N. Kudin, V. N. Staroverov, R. Kobayashi, J. Normand, K. Raghavachari, A. Rendell, J. C. Burant, S. S. Iyengar, J. Tomasi, M. Cossi, N. Rega, J. M. Millam, M. Klene, J. E. Knox, J. B. Cross, V. Bakken, C. Adamo, J. Jaramillo, R. Gomperts, R. E. Stratmann, O. Yazyev, A. J. Austin, R. Cammi, C. Pomelli, J. W. Ochterski, R. L. Martin, K. Morokuma, V. G. Zakrzewski, G. A. Voth, P. Salvador, J. J. Dannenberg, S. Dapprich, A. D. Daniels, Ö. Farkas, J. B. Foresman, J. V. Ortiz, J. Cioslowski and D. J. Fox, Gaussian09, Gaussian, Inc., Wallingford CT, 2009.

29 K. K. Irikura, R. D. Johnson and R. N. Kacker, J. Phys. Chem. A, 2005, 109, 8430.

30 G. Kresse and Furthmüller, Phys. Rev. B: Condens. Matter, 1996, 54, 11169.

31 R. Dovesi, R. Orlando, B. Civalleri, C. Roetti, V. R. Saunders and C. M. Zicovich-Wilson, Z. Kristallogr., 2005, 220, 571.

32 R. Dovesi, V. R. Saunders, C. Roetti, R. Orlando, C. M. F. P. Zicovich-Wilson, B. Civalleri, K. Doll, N. M. Harrison, I. J. Bush, P. D'Arco and M. Llunell, CRYSTAL09 User's Manual. In CRYSTAL09 User's Manual, University of Torino: Torino, 2009.

33 S. J. Clark, M. D. Segall, C. J. Pickard, P. J. Hasnip, M. I. J. Probert, K. Refson and M. C. Payne, Z. Kristallogr., 2005, 220, 567.

34 B. Civalleri, C. M. Zicovich-Wilson, L. Valenzano and P. Ugliengo, CrystEngComm, 2008, 10, 405.

35 M. D. King, W. D. Buchanan and T. M. Korter, Phys. Chem. Chem. Phys., 2011, 13, 4250.

36 C. Frayret, C. Masquelier, A. Villesuzanne, M. Morcrette and J.-M. Tarascon, Chem. Mater., 2009, 21, 1861.

37 C. Frayret, A. Villesuzanne, M. Pouchard and S. Matar, Int. J. Quantum Chem., 2005, 101, 826.

38 C. Frayret, A. Villesuzanne, N. Spaldin, E. Bousquet, J.N. Chotard, N. Recham and J.-M. Tarascon, Phys. Chem. Chem. Phys., 2010, 12, 15512.

39 J. Du and L. R. Corrales, J. Phys. Chem. B, 2006, 110, 22346. 40 M. Lontsi-Fomena, A. Villesuzanne, J. P. Doumerc, C. Frayret and M. Pouchard, Comput. Mater. Sci., 2008, 44, 53.

41 C. Gatti, TOPOND98 User's Manual, 1999. 
42 T. M. Krygowski, J. Chem. Inf. Model., 1993, 33, 70.

43 T. M. Krygowski and M. K. Cyrański, Chem. Rev., 2001, 101, 1385.

44 E. Matito, M. Duran and M. Sola, J. Chem. Phys., 2005, 122, 014109.

45 E. Matito, M. Duran and M. Sola, J. Chem. Phys., 2006, 125, 059901.

46 E. M. D. Keegstra, B.-H. Huisman, E. M. Paardekooper, F. J. Hoogesteger, J. W. Zwikker, L. W. Jenneskens, H. Kooijman, A. Schouten, N. Veldman and A. L. Spek, J. Chem. Soc. Perkin Trans., 1996, 2, 229.

47 H. Bock, S. Nick, W. Seitz, C. Naether and J. W. Z. Bats, Naturforsch. B Chem. Sci., 1996, 51, 153.

48 C. Frayret, E. I. Izgorodina, D. R. MacFarlane, A. Villesuzanne, A.-L. Barrès, O. Politano, D. Rebeix and P. Poizot, Phys. Chem. Chem. Phys., 2012, 14, 11398.

49 G. Bonnard, A.-L. Barrès, O. Mentré, D. G. Allis, C. Gatti, P. Poizot and C. Frayret, CrystEngComm, 2013, 15, 2809.

50 K. Molcanov, B. Kojic-Prodic, D. Babic, D. Zilic and B. Rakvin, CrystEngComm, 2011, 13, 5170.
51 K. Molcanov, B. Kojic-Prodic, D. Babic, D. Pajic, N. Novosel and K. Zadro, CrystEngComm, 2012, 14, 7958.

52 M. K. Aydinol, A. F. Kohan, G. Ceder, K. Cho and J. Joannopoulos, Phys. Rev. B: Condens. Matter, 1997, 56, 1354.

53 D. E. Hibbs, J. Overgaard and R. O. Piltz, Org. Biomol. Chem., 2003, 1, 1191.

54 E. Espinosa, E. Molins and C. Lecomte, Chem. Phys. Lett., 1998, 285, 170.

55 E. Espinosa, C. Lecomte and E. Molins, Chem. Phys. Lett., 1999, 300, 745.

56 C. Gatti, Z. Kristallogr., 2005, 220, 399.

57 U. Koch and P. L. A. Popelier, J. Phys. Chem., 1995, 99, 9747.

58 C. Frayret, A. Villesuzanne, M. Pouchard, F. Mauvy, J.M. Bassat and J.-C. Grenier, J. Phys. Chem. C, 2010, 114, 19062.

59 E. I. Izgorodina, U. L. Bernard, P. M. Dean, J. M. Pringle and D. R. Mac Farlane, Cryst. Growth Des., 2009, 9, 4834.

60 M. Palusiak and T. M. Krygowski, Chem.-Eur. J., 2007, 13, 7996. 